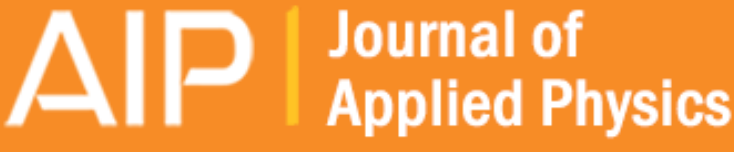

\section{Light helicity detection in MOS-based spin-photodiodes: An analytical model}

M. Cantoni and C. Rinaldi

Citation: Journal of Applied Physics 120, 104505 (2016); doi: 10.1063/1.4962204

View online: http://dx.doi.org/10.1063/1.4962204

View Table of Contents: http://scitation.aip.org/content/aip/journal/jap/120/10?ver=pdfcov

Published by the AIP Publishing

\section{Articles you may be interested in}

Enhancement of Spin-transfer torque switching via resonant tunneling

Appl. Phys. Lett. 105, 232410 (2014); 10.1063/1.4904408

Effect of the interface resistance of $\mathrm{CoFe} / \mathrm{MgO}$ contacts on spin accumulation in silicon

Appl. Phys. Lett. 100, 252404 (2012); 10.1063/1.4728117

Epitaxial Fe/MgO/Ge spin-photodiodes for integrated detection of light helicity at room temperature

J. Appl. Phys. 111, 07C312 (2012); 10.1063/1.3676243

High-temperature ultraviolet detection based on InGaN Schottky photodiodes

Appl. Phys. Lett. 99, 031115 (2011); 10.1063/1.3615291

Improving carrier transport and light emission in a silicon-nanocrystal based MOS light-emitting diode on silicon nanopillar array

Appl. Phys. Lett. 91, 093122 (2007); 10.1063/1.2778352

\section{High Energy Nanosecond Lasers}

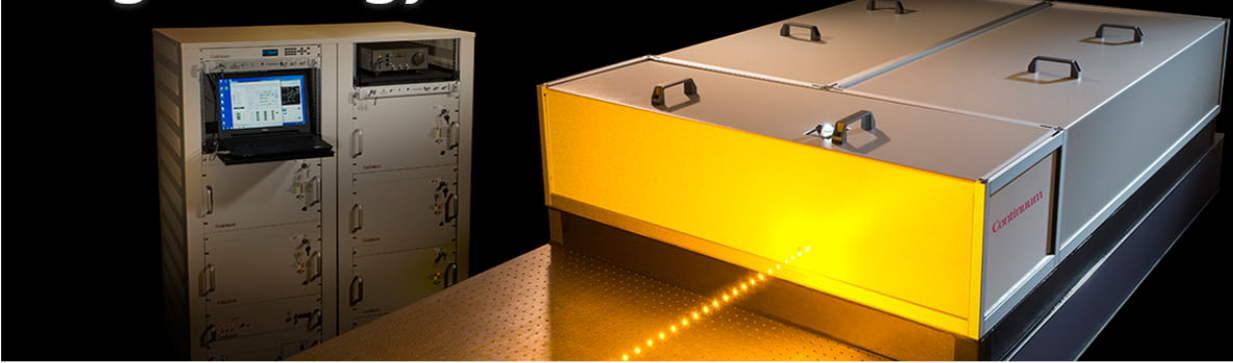




\title{
Light helicity detection in MOS-based spin-photodiodes: An analytical model
}

\author{
M. Cantoni ${ }^{\mathrm{a})}$ and C. Rinaldi \\ Dipartimento di Fisica, Politecnico di Milano, via G. Colombo 81, 20131 Milano, Italy
}

(Received 12 February 2016; accepted 22 August 2016; published online 8 September 2016)

\begin{abstract}
In a metal-oxide-semiconductor-based spin-photodiode, the helicity of an incoming light is efficiently converted into an electrical signal by exploiting (i) the helicity dependence of the degree of optical spin orientation for photogenerated carriers in the semiconductor and (ii) the spin-dependent tunneling transmission of the insulating barrier between the semiconductor and a ferromagnetic metal. Here, we propose a theoretical model for predicting the electrical response of the device to a circularly polarized light, by integrating the Fert-Jaffrès framework [A. Fert and H. Jaffrès, Phys. Rev. B 64, 184420 (2001)] with a helicity-dependent photo-generation term. A figure of merit, related to the variation of the electrical response to the switching of the light helicity from right to left, is defined, and its dependence on the constitutive parameters of the device (barrier resistivity and spin selectivity, semiconductor resistivity and spin diffusion length) is shown. Finally, a simple analytical formula for identifying the optimal resistance barrier leading to the maximum efficiency is found and experimentally validated on $\mathrm{Fe} / \mathrm{MgO} / \mathrm{Ge}$ spin-photodiodes. Published by AIP Publishing.
\end{abstract}

[http://dx.doi.org/10.1063/1.4962204]

\section{INTRODUCTION}

The potential of spin-optoelectronics relies on the possibility of manipulating and reading the photon helicity by acting on the magnetization of ferromagnetic electrodes in integrated devices, i.e., without the need of external optical elements. This is particularly appealing for information and communication technology applications such as data transmission cryptography, reconfigurable optical interconnects, optical switches, and modulators. ${ }^{1,2}$ Along the path of constructing a fully scalable technological platform for spin-optoelectronics, different elements are needed: optical guides or fibers preserving the state of circular polarization of light, ${ }^{3}$ spin-light-emitting-diodes (spin-LEDs), ${ }^{4-7}$ and spin-photodetectors (spin-PDs), ${ }^{8-11}$ converting electrical signals into photon helicity and vice versa. Despite the potential interest of such devices, a very limited number of theoretical models are available in the literature. Moreover, they typically employ quite complicated mathematics, ${ }^{12,13}$ while simple formulae relating the device performances to the physical parameters (materials, dimensions...) are lacking.

In this paper, we consider spin-photodetectors based on a Metal-Oxide-Semiconductor (MOS) structure, ${ }^{14-18}$ where the metal is a ferromagnet (FM) and the semiconductor (SC) presents a direct band-gap at the $\Gamma$-point of the electronic band structure (e.g., GaAs and Ge). This structure is highly suitable for the electrical reading of the light helicity because of (i) the helicity dependence of the degree of optical spin orientation for photo-generated carriers in the SC and (ii) the spin-dependent tunneling transmission of the insulating oxide barrier between the

\footnotetext{
a) Author to whom correspondence should be addressed. Electronic mail: matteo.cantoni@polimi.it
}

SC and the FM. The simplicity of the structure we propose, with respect to more complex templates such as those involving quantum wells ${ }^{10}$ or $\mathrm{p}-\mathrm{n}$ junctions, ${ }^{19}$ has different advantages: it allows for integration on Silicon (both $\mathrm{Ge}^{20}$ and $\mathrm{GaAs}^{21}$ can be epitaxially grown on $\mathrm{Si}$ substrates via proper buffer layers); it can be realized by either molecular beam epitaxy (MBE) (as the devices we show in Sec. V) and magnetron sputtering, since the spin filtering properties of the $\mathrm{MgO}$ barrier are essentially equivalent in the two cases $;{ }^{17}$ it can be described employing a simple formalism, with few relevant quantities and allowing an analytical solution, as we show below. The first two advantages, in particular, are well suitable for industrial exploitation of this technology, paving the way for a potential large-scale realization of CMOScompatible light polarization photodetectors. Moreover, these devices are fully integrated, because their operation does not need any external optical element (polarizers, quarter-wave plates) to be employed and can be scaled down to a micrometer scale, so that bi-dimensional arrays of spin-PDs can be realized in order to perform spatialresolved detection of light polarization. The potential applications of spin-PDs are extremely wide, from detection of molecule chirality in biology, to optical communications with circularly polarized light, and to three-statebased (left, right, and zero circular polarization) information technology.

The operating principle of the spin-PD can be schematized by a three-step process. First, spin polarized photo-carriers are generated in the SC by illuminating it with circularly polarized light; the photo-carrier spin polarization is determined by the optical selection rules acting in the $\Gamma$-point of the SC band-structure. ${ }^{22}$ Then, under the action of an electric field in the SC, produced by an external bias voltage, the photo-generated electrons 
and holes separate each other and move in opposite directions: in forward (reverse) bias, electrons (holes) move towards the barrier and undergo the tunneling. The transmission of the barrier depends on the relative orientation between the carrier spin polarization and the FM magnetization: this means that photocarriers suffer a different resistance for a spin parallel or antiparallel to the FM magnetization, and this finally leads to a modulation of the electrical response of the device (i.e., a different magnitude of the current at a fixed incident light intensity). Because the spin of the photo-carriers depends on the photon helicity, from the electrical response of the spin-PD is then possible to go back to the initial light polarization.

The conversion efficiency between the light helicity and the electrical response of the device may depend on many parameters, such as the thickness of the layers involved, the doping level of the SC, and the oxide barrier characteristics (resistance and spin selectivity). In this paper, we present a theoretical model (Secs. II and III) where the relationships between the conversion efficiency and the device parameters (Sec. IV) are ending up into a simple analytical formula giving the optimal parameter set for maximizing the spin-PD efficiency. Finally, an experimental validation is reported in Sec. V, where the relationship between the barrier thickness and the conversion efficiency in a $\mathrm{Fe} / \mathrm{MgO} / \mathrm{Ge}$-based spin$\mathrm{PD}$ is interpreted in the frame of our model.

\section{MODEL FOR SPIN-POLARIZED TRANSPORT WITH PHOTO-GENERATION}

Fig. 1(a) reports the scheme of our MOS device composed by (i) a ferromagnetic layer (FM) with thickness $z_{F M}$; (ii) an insulating barrier (B), that we treat as a zerothickness layer; and (iii) a semiconductor layer (SC) with thickness $z_{S C} . \Delta V$ is the voltage drop between the FM and the SC sides of the device. $J$ is the total current per unit area (from now on simply called current), flowing parallel to the $z$ axis. When the device works in forward bias (FB), that is $\Delta V>0$, electrons move from the $\mathrm{SC}$ to the FM, while holes move in the opposite direction. The device is illuminated by a circularly polarized light, propagating along the $z$ axis, coming from the FM side. Crossing the FM, light suffers differential absorption for left and right circular polarization due to Magnetic



FIG. 1. (a) Scheme of the MOS photodiode, composed by a ferromagnet (FM), an insulating barrier (B), and a semiconductor (SC), in the forward bias regime and (b) electrical setup employed for the constant voltage measurement mode (CVMM).
Circular Dichroism (MCD); anyway, this effect (that is very small, in the order of $0.5 \%$ for a wavelength of $1300 \mathrm{~nm}$ ) will not be considered in the present work, assuming that the light intensity reaching the SC is the same for both the polarizations. We note that it is quite easy, in experimental data, to disentangle the MCD contribution from the helicity-dependent transmission, employing the phenomenological model of Ref. 15, so that we can disregard the MCD effect in our analysis without loss of generality.

If the photon energy is larger than the SC band-gap, electron-hole pairs are photo-generated in the SC and separated by the electric field, due to the band bending in the depletion zone and/or to the external voltage $\Delta V$. In this paper, we make the same approximation of Ref. 23, assuming for simplicity that the SC bands are flat and neglecting any built-in voltage and Schottky barrier. This means that the SC, in our model, acts as a linear resistor. The currentvoltage characteristic of the device, that is essentially a tunnelling junction, is cubic; ${ }^{42,44}$ anyway, being always the photocurrent far smaller than the dc current $\left(J_{\mathrm{ph}} \ll J_{0}\right)$, it follows that the perturbation introduced by the illumination in terms of resistance is negligible, so that it can be thought as linear around the working point. Consequently, it is appropriate to treat the device as the series of three resistors (corresponding to FM, B, and SC, respectively), following the same approach of Fert and Jaffrès in Ref. 23. This simplification allows for solving analytically the model described below, leading to a mathematical expression for the spin filtering efficiency where all the parameter dependences are clearly defined. Moreover, we note that even if a fully general quantitative model should require band bending effects to be included, leading to a device structure typical of a diode (nonlinear current-voltage characteristics), our model works quite well for spin-PDs based on $\mathrm{MgO}$ barriers, because of Fermi level depinning at the interface between $\mathrm{MgO}$ and some semiconductors, such as $\mathrm{Ge}^{42}$ and GaAs. ${ }^{24}$

The total current flowing across the device $(J)$ is composed by two contributions: (i) a light-independent term $\left(J_{0}\right)$, produced by the application of the external voltage $\Delta V$, and (ii) the photocurrent $\left(J_{p h}\right)$ due to the light illumination, dependent on the light intensity reaching the SC and on the SC characteristics (absorption, quantum efficiency, and recombination). In a n-type $\mathrm{SC}, J_{0}$ is an electron current, while in $J_{p h}$ both electrons and holes are involved, because of the charge neutrality of the photo-generated pairs. As shown in Fig. 1(a), in forward bias photo-electrons move from the $\mathrm{SC}$ to the $\mathrm{FM}$, crossing $\mathrm{B}$ and thus experiencing spin filtering; the corresponding photo-generated holes, by contrary, move in the opposite direction, losing their spin information during their travel in the SC. In the following, we will focus our attention to the case of positive bias and to the electron current (from now on called $J$ ).

\section{A. Equation for spin-polarized transport with photo-generation in the semiconductor}

The model we present in this paper is based on the FertJaffrès $(\mathrm{FJ})$ model of the $\mathrm{FM} / \mathrm{B} / \mathrm{SC}$ template, ${ }^{23}$ to which we 
add a spin-dependent photo-generation term with nonuniform spatial distribution inside the SC, related to the light absorption profile. In the FJ model, the injection of a spin polarized current from a FM to a SC (or, more generally, to a non-magnetic material) is treated within the Valet-Fert (VF) framework, ${ }^{25}$ based on macroscopic transport equations derived from the Boltzmann equation when the spin relaxation is much slower than the momentum relaxation.

We start writing the continuity equation in the $\mathrm{SC}$ as follows:

$$
\frac{\partial n^{+(-)}}{\partial t}=\frac{1}{e} \frac{\partial J^{+(-)}}{\partial z}-\frac{n^{+(-)}-n^{-(+)}}{\tau_{s f}^{+(-)}} .
$$

The sign $+(-)$ indicates the absolute direction of the spin with respect to the $z$ axis (see Fig. $1\left(\right.$ a)). $n^{+(-)}$and $J^{+(-)}$ are the electron density and the current for $+(-)$ electrons at position $z$ in the SC. The left side and the first term in the right side of Eq. (1) correspond to the classical continuity equation: as a matter of fact, if we sum up Eq. (1) written for + electrons and for - electrons, we recover the classical continuity equation for the electron $\left(n=n^{+}+n^{-}\right)$and the current $\left(J=J^{+}+J^{-}\right)$densities. The second term in the right side of Eq. (1) accounts for the spin relaxation in the SC, with characteristic spin lifetime $\tau_{s f}^{+(-)}$. As discussed above and following $\mathrm{FJ}^{23}$ we consider a flat-band situation, i.e., any band bending in the SC depletion zone is neglected.

In a steady state condition, the electron densities $\left(n^{+}\right.$, $n^{-}$) are constant over time, so that Eq. (1) becomes

$$
\frac{1}{e} \frac{\partial J^{+(-)}}{\partial z}=\frac{n^{+(-)}-n^{-(+)}}{\tau_{s f}^{+(-)}} .
$$

This equation is equivalent to Eq. (10) in the VF work $^{25}$ that describes the one-dimensional macroscopic transport including spin relaxation. Employing the VF equation, we come to the following equation, where the electrochemical potentials $\left(\bar{\mu}^{+(-)}\right)$of the $+(-)$electrons have been introduced:

$$
\frac{n^{+(-)}-n^{-(+)}}{\tau_{s f}^{+(-)}}=\frac{\sigma^{+(-)}}{e^{2}} \frac{\bar{\mu}^{+(-)}-\bar{\mu}^{-(+)}}{l_{s f}^{+(-)^{2}}} .
$$

$\sigma^{+(-)}$is the electrical conductivity and $l_{s f}^{+(-)}$is the spin relaxation length in the SC for $+(-)$ electrons. $l_{s f}^{+(-)}$is related to the spin relaxation time $\tau_{s f}^{+(-)}$by the expression $l_{s f}^{+(-)}$ $=\sqrt{D^{+(-)} \tau_{s f}^{+(-)}}$, where $D^{+(-)}$is the diffusion coefficient for $+(-)$ electrons.

We now introduce the photocurrent in the continuity equation. The effective photo-generation of electrons $+(-)$ at position $z$ can be described by ${ }^{26}$

$$
G_{e f f}^{+(-)}(z)=\eta_{\sigma}^{+(-)} \exp \left(-\alpha_{L} z\right) .
$$

$\eta_{\sigma}^{+(-)}$is the photo-generation rate for $+(-)$electrons, that we assume constant over $z,{ }^{27}$ and $\alpha_{L}$ is the light absorption coefficient in SC. We assume that recombination of photoexcited electron and holes is included in the $\eta_{\sigma}^{+(-)}$coefficient that thus gives the net number of $+(-)$ photo-electrons per second that are available to electrical transport (see also Appendix A). We note that, because we are working in the forward bias regime, the contribution of spin polarized holes to the helicity dependent signal is null. As a matter of fact, holes drift away from the barrier and lose their spin polarization inside the SC, so that their contribution is not considered in our model.

The current density flowing into the photodiode is the sum of an unpolarised component $J_{0}$, generated by the voltage drop applied to the device, and a spin-polarized one $\left(J_{p h}\right)$ related to the photo-generation. Experimentally, $J_{0} \gg J_{p h}$ at each value of the bias (excluding $V_{\text {bias }}=0$ ), so that the recombination will be dominated by electrons from $J_{0}$ that do not contribute to the measured spin asymmetry. For this reason, we would expect a second order effect due to the recombination of photogenerated electrons with unpolarised holes. ${ }^{28}$ By including the photo-generation term (Eq. (4)) in the continuity equation (1), we obtain

$$
\frac{\partial n^{+(-)}}{\partial t}=\frac{1}{e} \frac{\partial J^{+(-)}}{\partial z}-\frac{n^{+(-)}-n^{-(+)}}{\tau_{s f}^{+(-)}}+\eta_{\sigma}^{+(-)} \exp \left(-\alpha_{L} z\right) .
$$

In a steady state condition and multiplying for $e^{2} \rho^{+(-)}$, Eq. (5) becomes

$$
e \rho^{+(-)} \frac{\partial J^{+(-)}}{\partial z}=\frac{\bar{\mu}^{+(-)}-\bar{\mu}^{-(+)}}{l_{s f}^{+(-)^{2}}}-e^{2} \rho^{+(-)} \eta_{\sigma}^{+(-)} \exp \left(-\alpha_{L} z\right),
$$

where we employed Eq. (3) and we defined the electrical resistivity $\rho^{+(-)}=1 / \sigma^{+(-)}$for $+(-)$electrons.

In addition to the continuity equation (1), $\mathrm{VF}^{25}$ considers the equivalent of the Ohm's law for $+(-)$ electrons (Eq. (11) in the VF work)

$$
J^{+(-)}=\frac{1}{e \rho^{+(-)}} \frac{\partial \bar{\mu}^{+(-)}}{\partial z}=\frac{1}{\rho^{+(-)}}\left[F \pm \frac{1}{e} \frac{\partial \Delta \mu}{\partial z}\right] .
$$

Following VF, we express the electrochemical potential for $+(-)$ electrons as $\bar{\mu}^{+(-)}=\bar{\mu} \pm \Delta \mu$, and we define $F$ as the gradient of $\bar{\mu}$ divided by $e$ (Eq. (12) in the VF work). ${ }^{25} \Delta \mu$ is the splitting (divided by two) between the electrochemical potentials of + and - electrons due to the spin accumulation in the SC. $F$ is equivalent to an electric field and takes into account the spin independent part of the electrochemical potential $(\bar{\mu})$.

By employing Eq. (7), we rewrite Eq. (6) as

$$
e \frac{\partial F}{\partial z} \pm \frac{\partial^{2} \Delta \mu}{\partial z^{2}}= \pm \frac{\Delta \mu}{l_{s f}^{+(-)^{2}}}-e^{2} \rho^{+(-)} \eta_{\sigma}^{+(-)} \exp \left(-\alpha_{L} z\right) .
$$

In a non-magnetic SC, the + and - resistivities are the same: $\rho^{+}=\rho^{-}=2 \rho_{S C}$. In a two current model, ${ }^{29} \rho_{S C}$ can be interpreted as the resistivity of the parallel between $\rho^{+}$ and $\rho^{-}$. The spin relaxation lengths are also equivalent: $l_{s f}^{+}=l_{s f}^{-}=\sqrt{2} l_{s f}^{S C}$, where $l_{s f}^{S C}$ is given by the relation $\left(1 / l_{s f}^{S C}\right)^{2}$ $=\left(1 / l_{s f}^{+}\right)^{2}+\left(1 / l_{s f}^{-}\right)^{2} \cdot 25$ 
We rewrite the photo-generation rate as $\eta_{\sigma}^{+(-)}$ $=\left(\bar{\eta}_{\sigma} \pm \Delta \eta_{\sigma}\right) / 2$, and we define the polarization of the photogenerated electron current as

$$
P_{\sigma}=\frac{\eta_{\sigma}^{+}-\eta_{\sigma}^{-}}{\eta_{\sigma}^{+}+\eta_{\sigma}^{-}}=\frac{\Delta \eta_{\sigma}}{\bar{\eta}_{\sigma}},
$$

$P_{\sigma}$ follows from the conservation of angular momentum and its value is determined by the selection rules relating the polarization state of the light $(\sigma)$ with the probability of exciting electron-hole pairs with a given spin direction $(+$ or - ) with respect to the $z$-component of the angular momentum $L=\sigma \hbar$. For example, in $\mathrm{GaAs}^{30}$ and $\mathrm{Ge}^{31,32}$ the maximum theoretical polarization is $P_{\sigma}=-0.5 \sigma$, where $\sigma=+1$ $(-1)$ means right (left) circular polarization, and can be achieved exciting the SC with a photon resonant with the direct gap at the $\Gamma$ point $(1.42 \mathrm{eV}$ for GaAs and $0.8 \mathrm{eV}$ for $\mathrm{Ge}$ at room temperature (RT)).

In order to separate the $F$ and $\Delta \mu$ contributions, we add and subtract Eq. (8) for + and for - electrons, leading to the following equations:

$$
\begin{gathered}
\frac{\partial F}{\partial z}=-e \rho_{S C} \bar{\eta}_{\sigma} \exp \left(-\alpha_{L} z\right), \\
\frac{\partial^{2} \Delta \mu}{\partial z^{2}}=\frac{\Delta \mu}{l_{s f}^{S C^{2}}}-e^{2} \rho_{S C} \Delta \eta_{\sigma} \exp \left(-\alpha_{L} z\right) .
\end{gathered}
$$

Their solutions are

$$
\begin{gathered}
F(z)=\frac{e \rho_{s c} \bar{\eta}_{\sigma}}{\alpha_{L}} \exp \left(-\alpha_{L} z\right)+A_{1}, \\
\Delta \mu(z)=A_{3} \exp \left(z / l_{s f}^{S C}\right)+A_{4} \exp \left(-z / l_{s f}^{S C}\right) \\
+\frac{l_{s f}^{2}}{1-\alpha_{L}^{2} l_{s f}^{S C^{2}}} e^{2} \rho_{S C} \Delta \eta_{\sigma} \exp \left(-\alpha_{L} z\right) .
\end{gathered}
$$

The electrochemical potentials $\bar{\mu}^{+(-)}$and the currents $J^{+(-)}$follow from Eqs. (12) and (13):

$$
\begin{gathered}
\bar{\mu}^{+(-)}(z)=-\frac{e^{2} \rho_{S C} \bar{\eta}_{\sigma}}{\alpha_{L}{ }^{2}} \exp \left(-\alpha_{L} z\right)+A_{1} e z+A_{2} \\
\pm A_{3} \exp \left(z / l_{s f}^{S C}\right) \pm A_{4} \exp \left(-z / l_{s f}^{S C}\right) \\
\pm \frac{l_{s f}^{S C^{2}}}{1-\alpha_{L}{ }^{2} l_{s f}^{S C^{2}}} e^{2} \rho_{S C} \Delta \eta_{\sigma} \exp \left(-\alpha_{L} z\right), \\
J^{+(-)}(z)=\frac{e \bar{\eta}_{\sigma}}{2 \alpha_{L}} \exp \left(-\alpha_{L} z\right)+\frac{A_{1}}{2 \rho_{S C}} \pm \frac{A_{3}}{2 e \rho_{S C} l_{s f}^{S C}} \exp \left(z / l_{s f}^{S C}\right) \\
\mp \frac{A_{4}}{2 e \rho_{S C} l_{s f}^{S C}} \exp \left(-z / l_{s f}^{S C}\right) \mp \frac{l_{s f}^{S C^{2}}}{1-\alpha_{L}^{2} l_{s f}^{S C^{2}}} \\
\times \frac{e \alpha_{L} \Delta \eta_{\sigma}}{2} \exp \left(-\alpha_{L} z\right) .
\end{gathered}
$$

$\bar{\mu}_{+(-)}=\bar{\mu} \pm \Delta \mu$ (Eq. (14)) is obtained by Eq. (13) and integrating $e F(z)$ (Eq. (12)) over $z$, according to the definition of $F .^{25} J^{+(-)}$(Eq. (15)) is obtained substituting $F$ (Eq. (12)) and $\Delta \mu$ (Eq. (13)) in Eq. (7). $A_{1}, A_{2}, A_{3}$, and $A_{4}$ are arbitrary constants, to be defined by the boundary conditions (BCs) of the problem, as described in Secs. II C and II D.

\section{B. Spin-polarized transport in the ferromagnet and across the barrier}

According to $\mathrm{FJ}^{23}$ the electrochemical potentials and the currents in the FM are

$$
\begin{gathered}
\bar{\mu}^{+(-)}(z)=\rho_{F M}\left(1-\beta^{2}\right) e \bar{J}_{z} \mp B_{1}(1 \pm \beta) \exp \left(z / l_{s f}^{F M}\right)+B_{2} \\
J^{+(-)}(z)=(1 \mp \beta) \frac{\bar{J}}{2} \mp \frac{B_{1}}{2 e \rho_{F M} l_{s f}^{F M}} \exp \left(z / l_{s f}^{F M}\right) .
\end{gathered}
$$

$\rho_{F M}^{+(-)}=2 \rho_{F M}(1 \pm \beta)$ is the FM resistivity for $+(-)$ electrons, ${ }^{25} l_{s f}^{F M}$ is the spin diffusion length in the FM; $\bar{J}$ is the electron current in the FM, constant along the FM thickness.

As in the FJ model, the barrier (B) at the FM/SC interface is treated as a zero-thickness layer, with a spindependent resistance for unit surface $r_{B}^{+(-)}=2 r_{B}(1 \pm \gamma){ }^{25} B_{1}$ and $B_{2}$ are arbitrary constants, to be defined by the boundary conditions in Secs. IIC and IID. By the way, we note that because both $A_{2}$ and $B_{2}$ play the role of additive constants in the electrochemical potentials (see Eqs. (14) and (16)), only one is actually meaningful (e.g., $B_{2}$ ), while the other one can be set to zero (e.g., $A_{2}=0$ ) without loss of generality. The parameters to be defined by the boundary conditions are then five: $A_{1}, A_{3}, A_{4}, B_{1}$, and $B_{2}$.

\section{Boundary conditions for the semiconductor}

The electron current in the SC is given by summing up Eq. (15) for $(+)$ and (-) electrons

$$
J(z)=J^{+}(z)+J^{-}(z)=\frac{e \bar{\eta}_{\sigma}}{\alpha_{L}} \exp \left(-\alpha_{L} z\right)+\frac{A_{1}}{\rho_{S C}} .
$$

Because of the photo-generation inside the SC, the electron current $J(z)$ varies exponentially along the SC with attenuation length $\lambda_{L}=\alpha_{L}{ }^{-1}$, where $\alpha_{L}$ is the light absorption coefficient in the SC. At a distance from the interface much larger than $\lambda_{L}\left(z \gg \lambda_{L}\right)$, only the light-independent term $J_{0}$ is present: no photo-electrons are created because light is completely attenuated, and those generated at a distance closer to the barrier will travel towards the barrier, in the forward bias regime, so that none of them will contribute to the current here. Assuming $z_{S C} \gg \lambda_{L}$, that is the SC length is much larger than the light attenuation length, the first boundary condition (BC) is thus $J\left(z_{S C}\right)=J_{0}$, from what it follows, from Eq. (18), $A_{1}=\rho_{S C} J_{0}$.

The second $\mathrm{BC}$ reflects the absence of spin accumulation $(\Delta \mu=0)$ at a distance from the interface much larger than the spin relaxation length $\left(z \gg l_{s f}^{S C}\right)$. If we assume $z_{S C} \gg l_{s f}^{S C}$, that is the SC length is much larger than the spin relaxation length, it follows $\Delta \mu\left(z_{S C}\right)=0$. From Eq. (13), recalling that $z_{S C} \gg \lambda_{L}$ as discussed above, we obtain $A_{3}=0$.

With $A_{1}=\rho_{S C} J_{0}$ and $A_{3}=0$, only three parameters remain to be given: $A_{4}, B_{1}$, and $B_{2}$.

\section{Matching conditions at the interface between the semiconductor and the ferromagnet}

According to Eq. (18), the current $J$ at the interface is continuous: $J\left(0^{+}\right)=J\left(0^{-}\right)$, where $z=0^{+}$and $z=0^{-}$correspond to the SC and FM sides of the interface, respectively 


$$
J_{0}+\frac{e \bar{\eta}_{\sigma}}{\alpha_{L}}=\bar{J} .
$$

As discussed in Sec. IIC, $J_{0}$ is the current in the SC far from the interface $\left(z \gg \alpha_{L}{ }^{-1}\right)$, while $\bar{J}$ is current in the FM, constant over length. $e \bar{\eta}_{\sigma} / \alpha_{L}$ (from now on called $J_{p h}$ ) can be interpreted as the contribution of the electrons photo-generated in the SC to the current at the interface (see also Appendix A).

Making the assumption of neglecting spin flip events at the interface, ${ }^{23} J^{+}$and $J^{-}$separately must be continuous in $z=0: J^{+(-)}\left(0^{+}\right)=J^{+(-)}\left(0^{-}\right)$. Because of the continuity of $J$ imposed above, only one of the two conditions (on $J^{+}$or $J^{-}$) is independent. For example, we consider the condition on $J^{+}$, that is $J^{+}\left(0^{+}\right)=J^{+}\left(0^{-}\right)$, and from Eqs. (15) and (17) it follows:

$$
\begin{aligned}
\frac{J_{0}}{2} & +\frac{\omega}{2 e r_{S C}} \bar{a}_{\sigma}-\frac{A_{4}}{2 e r_{S C}}-\frac{\omega}{2 e r_{S C}} \Delta a_{\sigma} \\
& =(1-\beta)\left[\frac{J_{0}}{2}+\frac{\omega}{2 e r_{S C}} \bar{a}_{\sigma}\right]-\frac{B_{1}}{2 e r_{F M}} .
\end{aligned}
$$

In Eq. (20), we used $A_{1}=\rho_{S C} J_{0}$ and $A_{3}=0$, we defined $r_{F M}=\rho_{F M} l_{s f}^{F M}, \quad r_{S C}=\rho_{S C} l_{s f}^{S C}, \quad$ and $\omega=\alpha_{L} l_{s f}^{S C}, \quad$ and we introduced

$$
\begin{gathered}
\Delta a_{\sigma}=\frac{e^{2} \rho_{S C} l_{s f}^{S C^{2}}}{1-\alpha_{L}{ }^{2} l_{s f}^{S C^{2}}} \Delta \eta_{\sigma}, \\
\bar{a}_{\sigma}=\frac{e^{2} \rho_{S C}}{\alpha_{L}{ }^{2}} \bar{\eta}_{\sigma} .
\end{gathered}
$$

While the + and - currents are continuous in $z=0$, the corresponding electrochemical potentials are not because of the voltage drop across the barrier: $\bar{\mu}^{+(-)}\left(0^{+}\right)-\bar{\mu}^{+(-)}\left(0^{-}\right)$ $=e r_{B}^{+(-)} J^{+(-)}(0)$. By using Eqs. (14) and (16) and remembering that $J^{+(-)}\left(0^{-}\right)=J^{+(-)}\left(0^{+}\right)$, we obtain the following conditions for + and - electrons:

$$
\begin{aligned}
& {\left[-\bar{a}_{\sigma} \pm A_{4} \pm \Delta a_{\sigma}\right]-\left[\mp B_{1}(1 \pm \beta)+B_{2}\right]} \\
& \quad=2 r_{B}(1 \pm \gamma) e\left[(1 \mp \beta)\left(\frac{J_{0}}{2}+\frac{\omega}{2 e r_{S C}} \bar{a}_{\sigma}\right) \mp \frac{B_{1}}{2 e r_{F M}}\right] .
\end{aligned}
$$

The solution of the system of Eqs. (20) and (23) for + and - electrons is reported in Appendix B.

\section{OPERATION OF THE SPIN-PHOTODIODE}

\section{A. Calculation of the voltage drop across the device}

When a current flows in the device, a voltage drop $\Delta V$ is produced between the FM $\left(z=-z_{F M}\right)$ and the SC $\left(z=z_{S C}\right)$ sides

$$
\Delta V=V\left(z_{S C}\right)-V\left(-z_{F M}\right)=\frac{\bar{\mu}\left(z_{S C}\right)-\bar{\mu}\left(-z_{F M}\right)}{e} .
$$

The spin independent parts of the electrochemical potentials, $\bar{\mu}\left(z_{S C}\right)$ and $\bar{\mu}\left(-z_{F M}\right)$, can be calculated from Eqs. (14) and (16)

$$
\begin{aligned}
\bar{\mu}\left(z_{S C}\right)= & \frac{\bar{\mu}^{+}\left(z_{S C}\right)+\bar{\mu}^{-}\left(z_{S C}\right)}{2} \\
= & \rho_{S C} e J_{0} z_{S C}-\bar{a}_{\sigma} \exp \left(-\alpha_{L} z_{S C}\right), \\
\bar{\mu}\left(-z_{F M}\right)= & \frac{\bar{\mu}^{+}\left(-z_{F M}\right)+\bar{\mu}^{-}\left(-z_{F M}\right)}{2} \\
= & -\rho_{F M}\left(1-\beta^{2}\right) e\left(J_{0}+\frac{e \bar{\eta}_{\sigma}}{\alpha_{L}}\right) z_{F M} \\
& -B_{1} \beta \exp \left(-z_{F M} / l_{s f}^{F M}\right)+B_{2} .
\end{aligned}
$$

Consequently, $\Delta V$ results

$$
\begin{aligned}
\Delta V= & \rho_{S C} J_{0} z_{S C}+\rho_{F M}\left(1-\beta^{2}\right)\left(J_{0}+\frac{e \bar{\eta}_{\sigma}}{\alpha_{L}}\right) z_{F M} \\
& -\frac{\bar{a}_{\sigma}}{e} \exp \left(-\alpha_{L} z_{S C}\right)+\frac{B_{1}}{e} \beta \exp \left(-z_{F M} / l_{s f}^{F M}\right)-\frac{B_{2}}{e},
\end{aligned}
$$

with the coefficients $B_{1}$ and $B_{2}$ given in Appendix B.

Assuming both the FM and the SC as semi-infinite (strictly, $z_{F M} \gg l_{s f}^{F M}$ and $z_{S C} \gg \alpha_{L}^{-1}$ ), the exponential terms in Eq. (27) can be neglected

$$
\Delta V=\bar{r}_{S C} J_{0}+\left(1-\beta^{2}\right) \bar{r}_{F M}\left(J_{0}+\frac{e \bar{\eta}_{\sigma}}{\alpha_{L}}\right)-\frac{B_{2}}{e},
$$

$\bar{r}_{S C}=\rho_{S C} z_{S C}$ and $\left(1-\beta^{2}\right) \bar{r}_{F M}=\left(1-\beta^{2}\right) \rho_{F M^{2}} z_{F M}$ are the resistances per unit area of the SC and of the FM, respectively. The latter is calculated as the parallel between $r_{F M}^{+}$ $=\rho_{F M}^{+} z_{F M}$ and $r_{F M}^{-}=\rho_{F M}^{-} z_{F M}$, with $\rho_{F M}^{+(-)}=2 \rho_{F M}(1 \pm \beta)$, according to the two current model. ${ }^{29}$ Then, $\bar{r}_{S C} J_{0}$ and $\left(1-\beta^{2}\right) \bar{r}_{F M}\left(J_{0}+e \bar{\eta}_{\sigma} / \alpha_{L}\right)$ are the voltage drops across the SC and the FM when crossed by currents $J_{0}$ and $J_{0}+J_{p h}$, respectively. The dependence from the degree of optical spin orientation for photo-generated carriers in the SC $\left(\Delta \eta_{\sigma}\right)$ enters only in the last term of Eq. (28), i.e., $-B_{2} / e$; the expression of $B_{2}$ is reported in Appendix B.

Before considering the general case (spin-PD illuminated by circularly polarized light), we start with trivial situations in order to verify the model, that is (a) without light and (b) with not polarized, or linearly polarized, light. After that, we will come to the more general case (c), with circularly polarized light.

(a) If no photo-excitation is present $\left(\bar{\eta}_{\sigma}=\Delta \eta_{\sigma}=0\right), B_{2}$ (see Appendix B) becomes

$$
B_{2}=-\frac{\left(1-\gamma^{2}\right) r_{B}^{2}+\left(1-2 \gamma \beta+\beta^{2}\right) r_{B} r_{F M}+r_{B} r_{S C}+\beta^{2} r_{S C} r_{F M}}{r_{F M}+r_{S C}+r_{B}} e J_{0}=-r^{*} e J_{0} .
$$


Correspondingly, $\Delta V$ is

$$
\Delta V=\left[\bar{r}_{S C}+\left(1-\beta^{2}\right) \bar{r}_{F M}+\frac{\left(1-\gamma^{2}\right) r_{B}^{2}+\left(1-2 \gamma \beta+\beta^{2}\right) r_{B} r_{F M}+r_{B} r_{S C}+\beta^{2} r_{S C} r_{F M}}{r_{F M}+r_{S C}+r_{B}}\right] J_{0},
$$

or by employing $r^{*}$ defined in Eq. (29)

$$
\Delta V=\left[\bar{r}_{S C}+\left(1-\beta^{2}\right) \bar{r}_{F M}+r *\right] J_{0}=r_{0} J_{0},
$$

$r_{0}$ can be defined as the resistance per unit area of the device in the absence of photo-excitation. If the FM is not magnetized, both the FM and B conductivities are spin independent (i.e., $\beta=\gamma=0$ ), then Eq. (30) reduces to $\Delta V=\left[\bar{r}_{S C}+\bar{r}_{F M}+r_{B}\right] J_{0}$ : the total resistance is just the series of the $\mathrm{SC}, \mathrm{FM}$, and B resistances, as reasonably expected.

(b) If the spin-PD is illuminated with either not polarized or linearly polarized light, ${ }^{33}$ photo-generation takes place $\left(\bar{\eta}_{\sigma} \neq 0\right)$ but without net degree of optical spin orientation $\left(\Delta \eta_{\sigma}=0\right)$. Taking $B_{2}$ from Appendix B, $\Delta V$ becomes

$$
\begin{aligned}
\Delta V & =\left[\bar{r}_{S C}+\left(1-\beta^{2}\right) \bar{r}_{F M}+r^{*}\right] J_{0}+\left\{\frac{\left(1-\gamma^{2}\right) r_{B}^{2}+\left(1-2 \gamma \beta+\beta^{2}\right) r_{B} r_{F M}+r_{B} r_{S C}+\beta^{2} r_{S C} r_{F M}}{r_{F M}+r_{S C}+r_{B}}+\frac{r_{S C}}{\omega}\right\} J_{p h} \\
& =r_{0} J_{0}+\left[r^{*}+\frac{r_{S C}}{\omega}\right] J_{p h} .
\end{aligned}
$$

With respect to case (a) (Eq. (31)), an additional voltage term appears because of the photo-generated carriers $\left(J_{p h}\right)$ crossing the device.

(c) Finally, if the spin-PD is illuminated by circularly polarized light $\left(\bar{\eta}_{\sigma} \neq 0, \Delta \eta_{\sigma} \neq 0\right), \Delta V$ becomes

$$
\begin{aligned}
\Delta V= & {\left[\bar{r}_{S C}+\left(1-\beta^{2}\right) \bar{r}_{F M}+r^{*}\right] J_{0}+\left(r^{*}+\frac{r_{S C}}{\omega}\right) J_{p h} } \\
& +\frac{\gamma r_{B}+\beta r_{F M}}{r_{F M}+r_{S C}+r_{B}}(\omega-1) \frac{\Delta a_{\sigma}}{e} .
\end{aligned}
$$

Rewriting $\Delta a_{\sigma}$ (see Eqs. (21) and (22)) as

$$
\Delta a_{\sigma}=P_{\sigma} \frac{\omega^{2}}{1-\omega^{2}} \bar{a}_{\sigma}=P_{\sigma} \frac{\omega}{1-\omega^{2}} r_{S C} e J_{p h},
$$

Eq. (33) can be expressed as

$$
\begin{aligned}
\Delta V= & {\left[\bar{r}_{S C}+\left(1-\beta^{2}\right) \bar{r}_{F M}+r^{*}\right] J_{0} } \\
& +\left[r^{*}+\frac{r_{S C}}{\omega}-r_{S C} \frac{\gamma r_{B}+\beta r_{F M}}{r_{F M}+r_{S C}+r_{B}} \frac{\omega}{1+\omega} P_{\sigma}\right] J_{p h} \\
= & r_{0} J_{0}+r_{\sigma} J_{p h} .
\end{aligned}
$$

Thus, the voltage drop $\Delta V$ is the sum of two contributions: (i) a helicity-independent term $r_{0} J_{0}$; and (ii) a helicitydependent term $r_{\sigma} J_{p h}$, where $r_{\sigma}$ (the term in square brackets multiplying $J_{p h}$ in Eq. (35)) is a function of the photo-current polarization $P_{\sigma}$.

\section{B. Constant current measurement mode (CCMM)}

We consider a spin-PD illuminated by a circularly polarized light with helicity $\sigma$ that produces in the SC an electron photocurrent $J_{p h}$ with degree of optical spin orientation $P_{\sigma}$. The photocurrent $J_{p h}=e \bar{\eta}_{\sigma} / \alpha_{L}$ depends on the light intensity (contained in the photo-generation rate $\bar{\eta}_{\sigma}$ ) and on the SC parameters $\left(\alpha_{L}, \bar{\eta}_{\sigma}\right)$ but is independent of the voltage drop $\Delta V$. We neglect any spurious effect of the light polarization (such as the magnetic circular dichroism in the FM layer). ${ }^{34}$

First, we discuss the case where we keep the total current $\bar{J}$ (see Eq. (19)) constant and fixed along the device. Because $J_{p h}$ depends on the SC and light properties only, during the photodiode operation $J_{0}=\bar{J}-J_{p h}$ is fixed too. We are interested in the behaviour of the voltage drop across the device ( $\Delta V^{\sigma}$, given by Eq. (35)) when the light polarization is switched from right $(\sigma=+1)$ to left $(\sigma=-1)$

$$
\begin{aligned}
\Delta\left(\Delta V^{\sigma}\right) & =\Delta V^{\sigma=-1}-\Delta V^{\sigma=+1} \\
& =r_{S C} \frac{\gamma r_{B}+\beta r_{F M}}{r_{F M}+r_{S C}+r_{B}} \frac{\omega}{1+\omega} \Delta P_{\sigma} J_{p h} \\
& =r_{S C}(S P)_{I} \frac{\omega}{1+\omega} \Delta P_{\sigma} J_{p h},
\end{aligned}
$$

where $\Delta P_{\sigma}=P_{\sigma=-1}-P_{\sigma=+1}$. We note that, for sake of symmetry, $P_{\sigma=-1}=-P_{\sigma=+1}$, so that $\Delta P_{\sigma}=2 P_{\sigma=-1}=-2 P_{\sigma=+1}$. In the following, for simplicity we will write $\Delta P_{\sigma}=2 P_{\sigma}$, assuming implicitly $\sigma=-1$ (left circular polarization).

We observe that $(S P)_{I}$ in Eq. (36) is formally equivalent to the spin polarization of the current at a $\mathrm{FM} / \mathrm{B} / \mathrm{SC}$ interface, in the absence of illumination, found by FJ (see Eq. (20) in Ref. 23). The same conclusions drawn by FJ apply also in this case: both $\gamma$ and $r_{B}$ must be different from zero in order to obtain a sizable spin-dependent signal $\Delta\left(\Delta V^{\sigma}\right)$; the larger is $r_{B}$ with respect to $r_{S C}$ and $r_{F M}$, the larger is $\Delta\left(\Delta V^{\sigma}\right)$. In the limit case $r_{B} \gg r_{S C}, r_{F M}$ we obtain $(S P)_{I} \approx \gamma$ and $\Delta\left(\Delta V^{\sigma}\right)$ achieves its maximum value 


$$
\Delta\left(\Delta V^{\sigma}\right) \approx 2 r_{S C} \gamma \frac{\omega}{1+\omega} P_{\sigma} J_{p h}=2 r_{S C} \gamma \frac{l_{s f}^{S C}}{\lambda_{L}+l_{s f}^{S C}} P_{\sigma} J_{p h}
$$

If $\lambda_{L} \ll l_{s f}^{S C}$ (as typically happens for photoelectrons excited above the SC gap at the $\Gamma$ point), ${ }^{16,18}$ the electrons are photo-generated close to the barrier: their path towards the latter is then very short, and the consequent depolarization is negligible. In this case, $\Delta\left(\Delta V^{\sigma}\right)$ becomes independent of $l_{s f}^{S C}$ and is directly proportional to the product $r_{S C} \gamma P_{\sigma}$. By contrary, if $\lambda_{L}>l_{s f}^{S C}$ (as for photoelectrons with energy resonant or very close to the SC gap at the $\Gamma$ point), ${ }^{16,18}$ the electrons are photo-generated far from the barrier, and thus can be easily depolarized while moving towards the barrier. In this case, $\Delta\left(\Delta V^{\sigma}\right)$ is reduced by the factor $l_{s f}^{S C} /\left(\lambda_{L}+l_{s f}^{S C}\right)<1$ and, in the limit $\lambda_{L} \gg l_{s f}^{S C}$, the helicity dependence of the voltage drop will be completely eliminated.

\section{Constant voltage measurement mode (CVMM)}

In this second measurement mode, the voltage drop $\Delta V$ is kept fixed and the total current $\left(\bar{J}^{\sigma}\right)$ is measured as a function of the light polarization $(\sigma)$. We define $\Delta J^{\sigma}=\bar{J}^{\sigma=-1}$ $-\bar{J}^{\sigma=+1}$ as the difference between the currents flowing in the device when it is illuminated by left $(\sigma=-1)$ and right $(\sigma=+1)$ circularly polarized light. Recalling that $\bar{J}^{\sigma}=J_{0}^{\sigma}$ $+J_{p h}$ and observing that $J_{p h}=e \bar{\eta}_{\sigma} / \alpha_{L}$ is independent of $\sigma$ and $\Delta V$, we obtain $\Delta J^{\sigma}=J_{0}^{\sigma=-1}-J_{0}^{\sigma=+1}$, where $J_{0}^{\sigma}$ comes from Eq. (35)

$$
J_{0}^{\sigma}=\frac{\Delta V-r_{\sigma} J_{p h}}{r_{0}}
$$

After some mathematics, $\Delta J^{\sigma}$ becomes

$$
\begin{aligned}
\Delta J^{\sigma}= & \frac{r_{\sigma=+1}-r_{\sigma=-1}}{r_{0}} J_{p h}=\frac{r_{S C}\left(\gamma r_{B}+\beta r_{F M}\right)}{\left(r_{F M}+r_{S C}+r_{B}\right)\left[\bar{r}_{S C}+\left(1-\beta^{2}\right) \bar{r}_{F M}\right]+\left(1-\gamma^{2}\right) r_{B}^{2}+\left(1-2 \gamma \beta+\beta^{2}\right) r_{B} r_{F M}+r_{B} r_{S C}+\beta^{2} r_{S C} r_{F M}} \\
& \times \frac{\omega}{1+\omega} 2 P_{\sigma} J_{p h} .
\end{aligned}
$$

Finally, we define the adimensional spin-dependent current asymmetry $A_{S F}=\Delta J^{\sigma} / J_{p h}$ that in the following will be taken as the figure of merit (or conversion efficiency) of the spin-PD:

$$
A_{S F}=\frac{r_{S C}\left(\gamma r_{B}+\beta r_{F M}\right)}{\left(r_{F M}+r_{S C}+r_{B}\right)\left[\bar{r}_{S C}+\left(1-\beta^{2}\right) \bar{r}_{F M}\right]+\left(1-\gamma^{2}\right) r_{B}^{2}+\left(1-2 \gamma \beta+\beta^{2}\right) r_{B} r_{F M}+r_{B} r_{S C}+\beta^{2} r_{S C} r_{F M}} \times \frac{\omega}{1+\omega} 2 P_{\sigma} .
$$

The optical spin orientation, the spin depolarization during propagation, and the spin filtering action of the barrier all contribute to $A_{S F}$. In Sec. IV, we will discuss the parameter dependence of $A_{S F}$ and which strategies should be taken in order to maximize it.

The circuit employed for measuring the current in the CVVM mode is reported in Fig. 1(b). A voltage generator keeps the voltage drop $\Delta V$ constant over the device. The current flowing in the photodiode (schematized as a resistor $R_{S D}$ ) gives rise, through the operational amplifier, to the output voltage $\Delta V_{O}=-R_{O} I=-R_{O}\left[I_{0}+I_{p h}(f)\right]$, where $R_{O}$ determines the gain of the amplifier. A lock-in technique is employed for measuring $\Delta V_{O}$, as discussed in Sec. $\mathrm{V}$, in order to improve the signal-to-noise ratio and to isolate the photocurrent contribution at frequency $f$.

\section{DISCUSSION}

As a prototype system for discussing the parameter dependence of $A_{S F}$ (Eq. (40)), we choose the epitaxial heterostructure $\mathrm{Fe} / \mathrm{MgO} / \mathrm{Ge}$ that has been extensively studied for spintronic applications ${ }^{35-41}$ also by the authors. ${ }^{14,15,42-46}$ In the following, we will consider an intrinsic Ge substrate with resistivity $\rho_{S C}=47 \Omega \mathrm{cm}$ (accidentally n-doped), illuminated by monochromatic light with a wavelength of $1300 \mathrm{~nm}$ (coincident with one of the transmission windows of
Si-based optical fibers). The corresponding photon energy is $0.95 \mathrm{eV}$, close to the direct gap at the $\Gamma$ point of the Ge band structure $(0.8 \mathrm{eV})$. We note that the flat-band approximation is reasonable with such a Ge substrate, being the built-in voltage very small $\left(V_{\mathrm{BI}} \sim 0.12 \mathrm{eV}\right.$, calculated as the voltage to which corresponds a zero photocurrent in Ref. 15) and the depletion width very large $(\sim 5 \mu \mathrm{m})$. Moreover, the $\mathrm{I}-\mathrm{V}$ curve measured on a tunnelling junction of comparable area (data not shown) is cubic, indicating that the main transport mechanism is tunnelling through the $\mathrm{MgO}$ barrier.

For the polarization of photo-generated electrons, we assume $P_{\sigma}=0.5$ as for direct gap excitation, according to the optical selection rules holding at the $\Gamma$ point. ${ }^{47} \mathrm{We}$ note that this value has also been experimentally confirmed, within a tolerance of $\pm 10 \%$, by different experiments, both spin polarized photoemission ${ }^{48,49}$ and photoluminescence. ${ }^{50}$ Anyway, considering that $P_{\sigma}$ appears in Eq. (40) only as a multiplying factor, a different value of this parameter would only reduce the conversion efficiency by dividing for a constant factor, while the remaining part of the discussion will be unaffected.

For the electron spin relaxation time, we assume $\tau_{s f}^{S C}$ $=100$ ps, extrapolated from Ref. 51 at room temperature. Correspondingly, employing a diffusion coefficient $D$ $=0.01035 \mathrm{~m}^{2} \mathrm{~s}^{-1}$ for electrons, ${ }^{52}$ the spin relaxation length results $l_{s f}^{S C}=\sqrt{D \tau_{s f}^{S C}}=1.02 \mu \mathrm{m}$. Consequently, we obtain 
$r_{S C}=\rho_{S C} l_{s f}^{S C}=4.78 \times 10^{-7} \Omega \mathrm{m}^{2}$ and, employing a light attenuation length $\lambda_{L}=1.25 \mu \mathrm{m},{ }^{53} \omega=l_{s f}^{S C} / \lambda_{L}=0.814$.

Note that the only effect of the light attenuation length $\lambda_{L}$ on $A_{S F}$ (see Eq. (40)) is via the pre-factor $\omega /[1+\omega]$ $=l_{s f}^{S C} /\left[\lambda_{L}+l_{s f}^{S C}\right]$ that affects the magnitude of $A_{S F}$ but not its dependence on $r_{B}$ and $\gamma$. If $\lambda_{L} \ll l_{s f}^{S C}(\omega \gg 1)$, the degree of optical spin orientation of the photo-generated electrons is preserved from the generation points to the barrier: this condition clearly gives a maximum in $A_{S F}(\omega /[1+\omega] \approx 1)$. If $\lambda_{L} \gg l_{s f}^{S C}(\omega \ll 1)$, instead, the degree of optical spin orientation of the photo-generated electrons is almost completely lost before coming to the barrier, so that $A_{S F}$ tends to zero $(\omega /[1+\omega] \rightarrow 0)$. For the present case, $\omega=l_{s f}^{S C} / \lambda_{L}=0.814$ so that $\omega /[1+\omega]=0.45$.

For Fe, we employ $\beta=0.41$ as bulk resistance asymmetry, ${ }^{54} l_{s f}^{F M}=8.5 \mathrm{~nm}$ as spin diffusion length, ${ }^{55}$ and $\rho_{\mathrm{FM}}=4$ $\times 10^{-6} \Omega \mathrm{cm}$ as resistivity, so that $r_{F M}=\rho_{F M} l_{s f}^{F M}=3.4$ $\times 10^{-16} \Omega \mathrm{m}^{2}$

For the barrier, we employ $r_{B}=5 \times 10^{-6} \Omega \mathrm{m}^{2}$ and $\gamma=0.35$ (see Ref. 15). ${ }^{56}$

The device we consider has a circular area, with $750 \mu \mathrm{m}$ diameter (see the inset of Fig. 7 for a top view of the device); the $\mathrm{Ge}$ and $\mathrm{Fe}$ thicknesses are, respectively, $z_{S C}=20 \mu \mathrm{m}$ (Ref. 57) and $z_{F}=10 \mathrm{~nm}$, holding to $\bar{r}_{S C}=\rho_{S C} z_{S C}=9.4 \times 10^{-7} \Omega$ $\mathrm{m}^{2}$ and $\bar{r}_{F M}=\rho_{F M} z_{F M}=4 \times 10^{-16} \Omega \mathrm{m}^{2}$. An estimation of the photogeneration rate $\left(\bar{\eta}_{\sigma}\right)$ can be obtained from photocurrent experimental data and Eq. (19): with $J_{p h} \sim 40 \mathrm{~A} / \mathrm{m}^{2}$ at $0.4 \mathrm{~V}$ bias (corresponding to $I_{p h} \sim 20 \mu \mathrm{A}$ ), we find $\bar{\eta}_{\sigma}=\alpha_{L} J_{p h} /$ $e \sim 2 \times 10^{26}$ electrons $/ \mathrm{m}^{3}$ s. From Eqs. (29), (31), and (35) we calculate $r^{*}=4.44 \times 10^{-6} \Omega \mathrm{m}^{2}, r_{0}=1.38 \times 10^{-5} \Omega \mathrm{m}^{2}, r_{\sigma+}$ $=5.06 \times 10^{-6} \Omega \mathrm{m}^{2}$, and $r_{\sigma-}=4.99 \times 10^{-6} \Omega \mathrm{m}^{2}$.

Assuming a voltage drop $\Delta V=0.4 \mathrm{~V}$ and working in the CVMM mode (see Sec. III C), we can evaluate the dc densities in the (a)-(c) cases discussed in Sec. III A:

(a) $J_{0}=2.890 \times 10^{4} \mathrm{~A} / \mathrm{m}^{2}\left(I_{0} \sim 13 \mathrm{~mA}\right)$ if no photo-excitation is present;

(b) $J_{0}=2.889 \times 10^{4} \mathrm{~A} / \mathrm{m}^{2}$ if the device if illuminated by not polarized, or linearly polarized, light with $J_{p h}=40 \mathrm{~A} / \mathrm{m}^{2}$ $\left(I_{p h} \sim 20 \mu \mathrm{A}\right.$ ); we note that the difference with case (a) is very small because the photocurrent is a perturbation with respect to the dc $\left(J_{p h} / J_{0} \sim 0.1 \%\right)$;

(c) $J_{0}^{\sigma=+1}=2.88851 \times 10^{4} \mathrm{~A} / \mathrm{m}^{2}$ and $J_{0}^{\sigma=-1}=2.88853 \times 10^{4}$ $\mathrm{A} / \mathrm{m}^{2}$ if the device is illuminated by circularly polarized light with right $(\sigma=+1)$ or left $(\sigma=-1)$ circular polarization. $\Delta J^{\sigma}$ results $\sim 2 \times 10^{-1} \mathrm{~A} / \mathrm{m}^{2}(\sim 90 \mathrm{nA})$, leading to a conversion efficiency $A_{S F}=\Delta J^{\sigma} / J_{p h} \sim 0.5 \%$.

In Fig. 2 are reported, as a function of the voltage drop $\Delta V$, the current density without photo-excitation $\left(J_{0}\right.$, black curve and left scale) and the difference between the current densities after illumination by left $(\sigma=-1)$ and right $(\sigma=+1)$ circularly polarized light $\left(\Delta J^{\sigma}\right.$, red curve and right scale). For the photocurrent $\left(J_{p h}\right)$, we assumed a linear relation between $\Delta V$ and $J_{p h}$. We note that this is strictly true in the flat band condition: in this case, the electric field in the $\mathrm{SC}$ is the only driving force for separating the electron-hole pairs, and thus it is reasonable to assume, at least at the first order, a proportionality with the photocurrent intensity. In



FIG. 2. Current density without photo-excitation $\left(J_{0}\right.$, black curve and left scale) and current density asymmetry between left $(\sigma=-1)$ and right $(\sigma=+1)$ circularly polarized light $\left(\Delta J^{\sigma}\right.$, red curve and right scale). In the inset are reported the current densities after illumination by left (top red curve) and right (bottom blue curve) circularly polarized light, and by comparison the corresponding curve for unpolarised, or linearly polarized, light (black dashed curve), around the working point of the device $(\Delta V=0.4 \mathrm{~V})$.

the inset are reported the current densities after illumination by left (top red curve) and right (bottom blue curve) circularly polarized light, and by comparison the corresponding curve for unpolarised, or linearly polarized, light (black dashed curve), around the working point of the device $(\Delta V=0.4 \mathrm{~V})$.

Before going on, it is worthwhile to discuss, in our system, the eventuality of an electric-field dependence of spin diffusion in the SC, as predicted by Yu and Flatté (see Ref. 58). In order to evaluate this effect, we estimate the maximum electric field (represented by $F(z)$, see Eqs. (7), (10), and (12)) in the SC: at the interface, it results $F(z=0)$ $=e \rho_{S C} \bar{\eta}_{\sigma} / \alpha_{L}+\rho_{S C} J_{0}=\rho_{S C}\left(J_{0}+J_{p h}\right)=1.36 \times 10^{4} \mathrm{~V} / \mathrm{m}$. The high-field regime for spin transport in SCs, in which the decay length of spin polarization is no longer the spin relaxation length, is defined in Ref. 58 by the condition $E>E_{\mathrm{C}}$, where $E$ is the electric field $\left(F=1.36 \times 10^{4} \mathrm{~V} / \mathrm{m}\right.$, with our notation) and $E_{\mathrm{C}}$ is a critical value given by the relation $e E_{C} / k_{B} T=1 / l_{s f}^{S C} . T$ is the temperature $(300 \mathrm{~K})$ and $k_{B}$ is the Boltzmann constant. $E_{\mathrm{C}}$ results $2.54 \times 10^{4} \mathrm{~V} / \mathrm{m}$, larger than $E$ : the influence of the electric field on the spin diffusion regime can then be neglected, and the decay length of spin polarization in $\mathrm{SC}$ can be represented by the spin relaxation length $l_{s f}^{S C}$. 59

\section{A. Current asymmetry vs. barrier asymmetry $\left(A_{S F}\right.$ VS. $\left.\gamma\right)$}

In Fig. 3, we report the color plot of $A_{S F}$, obtained by Eq. (40) with $\tau_{s f}^{S C}=100 \mathrm{ps}\left(l_{s f}^{S C} \sim 1 \mu \mathrm{m}\right)$, as a function of the barrier resistance $\left(r_{B}\right)$ and asymmetry $(\gamma)$. In the bottom panel is shown the line profile of $A_{S F}$ vs. $\gamma$ corresponding to $r_{B}=5 \times 10^{-5} \Omega \mathrm{m}^{2} \cdot{ }^{42}$ Before to continue, it is helpful to make some approximations. As the values reported above suggest, $r_{F M}$ can be reasonably neglected when compared with $r_{S C}$ and $\bar{r}_{F M}$ when compared to $\bar{r}_{S C}$. This is a quite general situation for ferromagnetic metals, so that the approximations $r_{F M} / r_{S C} \approx 0$ and $\bar{r}_{F M} / \bar{r}_{S C} \approx 0$ can be always made without loss of generality. Moreover, apart from the special 


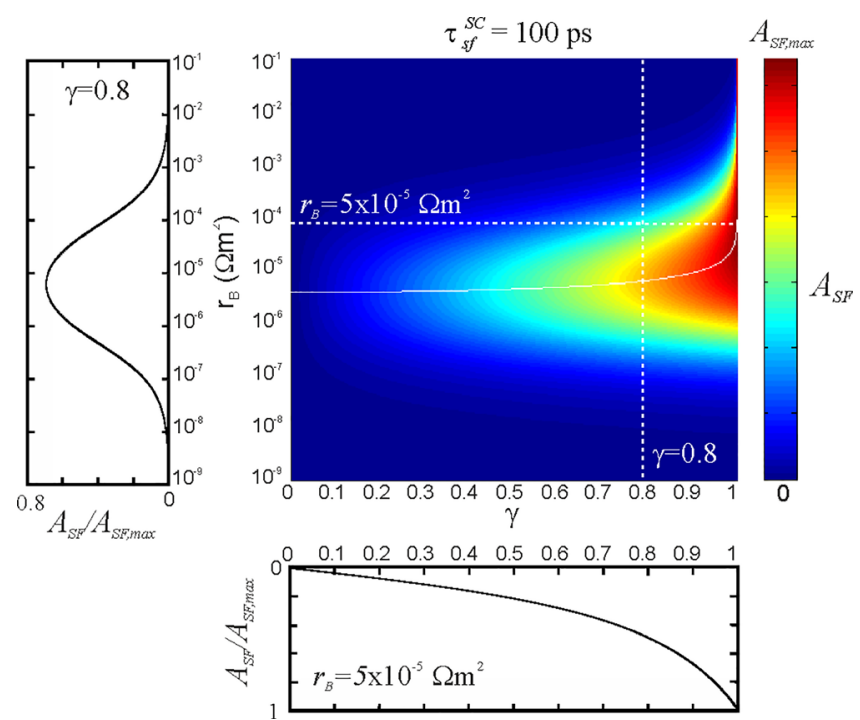

FIG. 3. Calculated conversion efficiency $\left(A_{S F}\right)$ of the spin-photodiode as a function of the barrier resistance $\left(r_{B}\right)$ and asymmetry $(\gamma)$. A spin relaxation time $\tau_{s f}^{S C}=100 \mathrm{ps}$ has been assumed. In the left and bottom panels are reported the cross sections for $\gamma$ and $r_{B}$ fixed, respectively.

case in which the barrier is absent $\left(r_{B}=0\right)$, that will be discussed in detail below, also the tunnelling barrier resistance $r_{B}$ is typically some orders of magnitude larger than $r_{F M}$, so that we can also assume $r_{F M} / r_{B} \approx 0$ and $\beta r_{F M} / \gamma r_{B} \approx 0$. With these approximations, $A_{S F}(\gamma>0)$ (Eq. (40)) results, neglecting $\beta r_{F M}$ at the numerator ${ }^{60}$

$$
A_{S F}(\gamma>0) \approx \frac{\gamma r_{B} r_{S C}}{\bar{r}_{S C}\left(r_{S C}+r_{B}\right)+\left(1-\gamma^{2}\right) r_{B}^{2}+r_{B} r_{S C}} \frac{\omega}{1+\omega} 2 P_{\sigma} .
$$

In the special case $\gamma=1$, the current is $100 \%$ spin polarized by the barrier (only + electrons cross the barrier), and $A_{S F}$ results

$$
\begin{aligned}
A_{S F}(\gamma=1) & \approx \frac{r_{B} r_{S C}}{\bar{r}_{S C}\left(r_{S C}+r_{B}\right)+r_{B} r_{S C}} \frac{\omega}{1+\omega} 2 P_{\sigma} \\
& =\frac{1}{1+\frac{z_{S C}}{l_{s f}^{S C}}+\frac{\bar{r}_{S C}}{r_{B}}} \frac{\omega}{1+\omega} 2 P_{\sigma} .
\end{aligned}
$$

The absolute maximum of $A_{S F}$ is attained for $\gamma=1$ and $r_{B} \gg \bar{r}_{S C}$

$$
A_{S F, \max } \approx \frac{1}{1+\frac{z_{S C}}{l_{s f}^{S C}}} \frac{\omega}{1+\omega} 2 P_{\sigma}=\frac{l_{s f}^{S C}}{l_{s f}^{S C}+z_{S C}} \frac{l_{s f}^{S C}}{l_{s f}^{S C}+\lambda_{L}} 2 P_{\sigma} .
$$

\section{B. Current asymmetry vs. barrier resistance (AsF vs. $\left.r_{B}\right)$}

In the left panel of Fig. 3 is reported the line profile of $A_{S F}$ vs. $r_{B}$ corresponding to $\gamma=0.8$ and $\tau_{s f}^{S C}=100 \mathrm{ps}\left(l_{s f}^{S C} \sim 1 \mu \mathrm{m}\right)$. When $r_{B} \ll r_{S C}, A_{S F}$ (Eq. (40)) can be approximated to

$$
A_{S F}\left(r_{B} \ll r_{S C}\right) \approx \frac{\gamma r_{B}+\beta r_{F M}}{\bar{r}_{S C}} \frac{\omega}{1+\omega} 2 P_{\sigma} .
$$

In the absence of the barrier $\left(r_{B}=0\right)$, the numerator of Eq. (44) is proportional to $\beta r_{F M}$, smaller than $\gamma r_{B}$ as discussed above, so that $A_{S F}$ is minimum. This is an intuitive result, being the presence of the spin dependent barrier $\left(r_{B} \neq 0, \gamma \neq 0\right)$ necessary for exploiting sizable spin dependent effects, as already pointed out by $\mathrm{FJ}^{23}$ and other authors. $^{61,62}$

When $r_{B} \gg r_{S C}$, instead, we have $\gamma r_{B} \gg \beta r_{F M}$ and $A_{S F}$ (Eq. (40)) becomes

$$
A_{S F}\left(r_{B} \gg r_{S C}\right) \approx \frac{\gamma}{1+\frac{\bar{r}_{S C}}{r_{S C}}+\left(1-\gamma^{2}\right) \frac{r_{B}}{r_{S C}}} \frac{\omega}{1+\omega} 2 P_{\sigma}
$$

In the asymptotic case $r_{B} \rightarrow \infty$, we have $A_{S F} \rightarrow 0$, apart from the case $\gamma=1$ that has been discussed above (see Eq.(43)). This result can be justified as follows. At fixed $\gamma$, for low values of $r_{B}$, the impact of spin filtering is negligible and the different spin populations optically injected in the $\mathrm{SC}$ do not produce a sizable electrical signal. An increase in $r_{B}$ favors electrical detection, and consequently the current asymmetry $\Delta J^{\sigma}$ increases. However, according to the FJ theory of spin injection in $\mathrm{SCs},{ }^{23}$ large $r_{B}$ values also produce a sizable spin splitting of the electrochemical potentials in the SC simply due to dc current. In these conditions, spin polarized photo-generated electrons suffer from a relevant depolarization during their motion towards the barrier and $\Delta J^{\sigma}$ decreases, leading to small values of $A_{S F}$. As a consequence, there is an optimum value of $r_{B}$ ensuring the maximization of $A_{S F}$ (the calculation is reported in Appendix C)

$$
\overline{r_{B}}=\rho_{S C} \sqrt{\frac{z_{S C} l_{s f}^{S C}}{1-\gamma^{2}}} .
$$

The locus $\overline{r_{B}}(\gamma)$ is reported in Fig. 3 (continuous white line).

In Fig. 4 is reported the helicity-dependent resistance $r_{\sigma}$ (Eq. (35)), normalized to the semiconductor resistance $r_{S C}$, as a function of the ratio $r_{B} / r_{S C}$ for $P_{\sigma}=-0.5$ (red line), 0 (black line), and +0.5 (blue line), corresponding to right, null (linear), and left circular polarization. When $r_{B} \ll r_{S C}$, we have

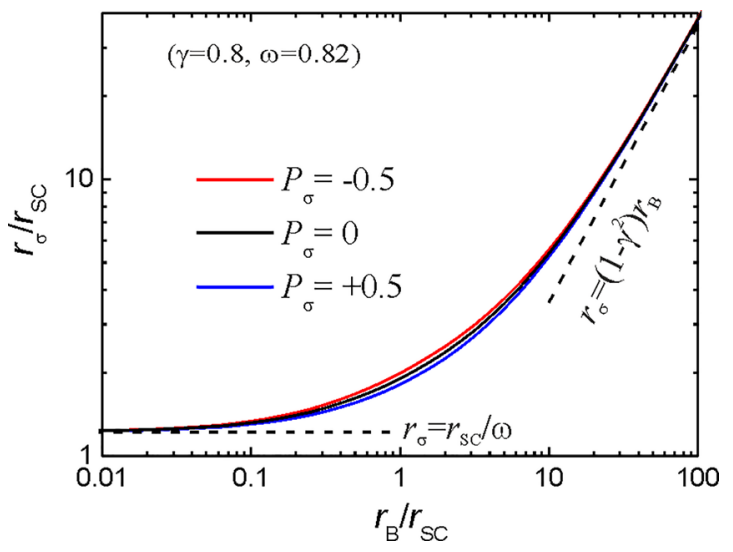

FIG. 4. Helicity-dependent resistance $r_{\sigma}$ (see Eq. (35) in the text) as a function of the ratio $r_{B} / r_{S C}$, for $P_{\sigma}=-0.5$ (red line), 0 (black line), and +0.5 (blue line). $\gamma=0.8$ and $\omega=l_{s f}^{S C} / \lambda_{L}=0.82$ are assumed. 


$$
\frac{r_{\sigma}}{r_{S C}}=\frac{1}{\omega}+\frac{r_{B}}{r_{S C}}\left(1-\gamma \frac{\omega}{1+\omega} P_{\sigma}\right),
$$

that in the limit $r_{B} / r_{S C} \rightarrow 0$ leads to

$$
r_{\sigma}=\frac{r_{S C}}{\omega}=\rho_{S C} \lambda_{L},
$$

$r_{\sigma}$ acts as an "effective resistance," leading to the voltage drop $r_{\sigma} J_{p h}$ (see Eq. (35)) seen by photoelectrons produced in $\mathrm{SC}$ according to a spatial distribution with characteristic length $\lambda_{L}$. Anyway, when $r_{B} / r_{S C} \rightarrow 0, r_{\sigma}$ is constant and the helicity dependence is lost.

When $r_{B} \gg r_{S C}$, instead, we have

$$
\frac{r_{\sigma}}{r_{S C}}=\frac{\left(1-\gamma^{2}\right) r_{B}}{r_{S C}}+\frac{1}{\omega}-\gamma \frac{\omega}{1+\omega} P_{\sigma},
$$

that in the limit $r_{B} / r_{S C} \rightarrow \infty$ leads to

$$
r_{\sigma}=\left(1-\gamma^{2}\right) r_{B}
$$

Even in this case, $r_{\sigma}$ is constant and the helicity dependence is lost. While in the limit $r_{B} / r_{S C} \rightarrow 0$, the voltage drop $r_{\sigma} J_{p h}$ (see Eq. (35)) was ascribed to the SC resistance seen by photoelectrons during their motion towards the barrier (Eq. 48)), in the limit $r_{B} / r_{S C} \rightarrow \infty$ it is due to the barrier resistance itself (Eq. (50)); in both the cases, there is no dependence on $P_{\sigma}$, so that $\Delta J_{\sigma}$ (Eq. (39)) and $A_{S F}$ (Eq. (40)) become zero, as shown in Fig. 3 (left panel).

\section{Current asymmetry vs. spin relaxation time ( $A_{\mathrm{SF}}$ vs. $\tau_{s f}^{S C}$ )}

In Fig. 5, we report the color plots of $A_{S F}$ for three different electron spin relaxation times: $\tau_{s f}^{S C}=1$ ps (panel (a)), 100 ps (panel (b), the same of Fig. 3), and 10 ns (panel (c)), corresponding to $l_{s f}^{S C} \sim 0.1 \mu \mathrm{m}, 1 \mu \mathrm{m}$, and $10 \mu \mathrm{m}$, respectively. In each panel, the optimal resistance barrier $\bar{r}_{B}(\gamma)$ is shown (continuous white line). In Fig. 6 is reported $\bar{r}_{B}$ vs. $\tau_{s f}^{S C}$ and $l_{s f}^{S C}$ with $\gamma=0.8$ (continuous black line): according to Eq. (46), $\bar{r}_{B}$ increases with $l_{s f}^{S C}$. The maximum value of $A_{S F}$ (Eq. (43)) also increases with $\tau_{s f}^{S C}\left(l_{s f}^{S C}\right)$, as shown in Fig. 6 (continuous red and dashed blue lines). As discussed above, a larger $\tau_{s f}^{S C}\left(l_{s f}^{S C}\right)$ means that the photo-electrons generated in the SC have a larger probability to cross the device and get to the barrier without losing their spin polarization: correspondingly, $A_{S F}$ is larger.

We note that, from Eq. (43), how large $l_{s f}^{S C}$ depends on the comparison with two other characteristic lengths: the SC length $z_{S C}$ and the light absorption length $\lambda_{L}$. The latter depends both on the SC properties and the light wavelength. In Fig. 6, we report $A_{S F, \max }$ vs. $\tau_{s f}^{S C}\left(l_{s f}^{S C}\right)$ for two different light absorption lengths, $1.25 \mu \mathrm{m}$ (red line) and $12.5 \mu \mathrm{m}$ (blue dashed line), corresponding to $1300 \mathrm{~nm}$ and $1550 \mathrm{~nm}$ light wavelengths, respectively. ${ }^{63}$

Note that we employ the same approximation followed in Ref. 31, where Ge is treated like a direct semiconductor for what concern selection rules. In fact, indirect phononassisted absorption processes are much weaker than those at the direct gap (about three to four orders of magnitudes from
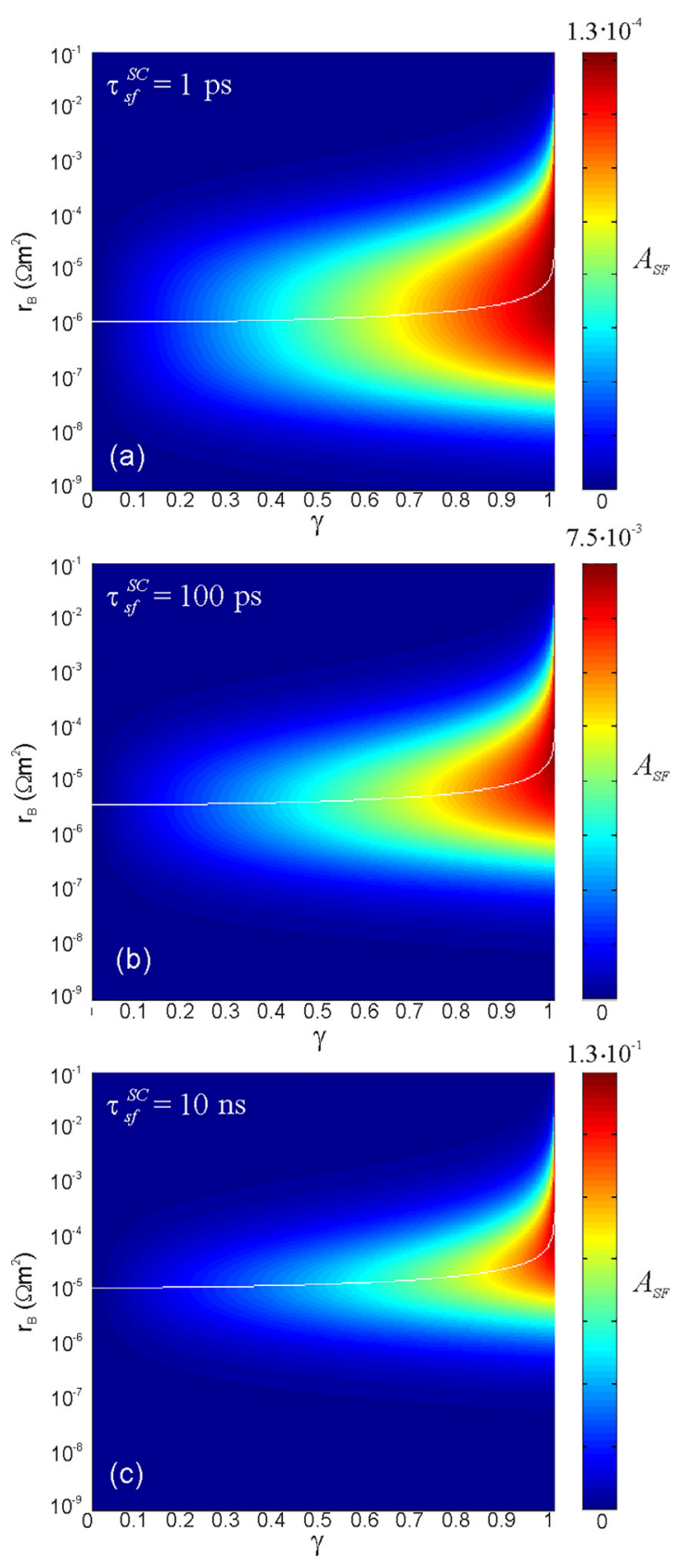

FIG. 5. Calculated conversion efficiency $\left(A_{S F}\right)$ of the spin-photodiode as a function of the barrier resistance $\left(r_{B}\right)$ and asymmetry $(\gamma)$ for three different electron spin relaxation times: $\tau_{s f}^{S C}=1$ ps (panel (a)), 100 ps (panel (b)), and $10 \mathrm{~ns}$ (panel (c)). The optimal resistance barrier $\left(\bar{r}_{B}\right.$ vs. $\left.\gamma\right)$ is indicated in each panel (continuous white line).

indirect transitions from $\Gamma$ to $\mathrm{L}$ at $h \nu=0.66 \mathrm{eV}$ to direct transitions at $\Gamma$ with $h \nu=0.8 \mathrm{eV}$ (Ref. 63)), so that indirect transitions can be neglected.

As expected from the discussion above, and assuming the same value for $\tau_{s f}^{S C}\left(l_{s f}^{S C}\right)$ at the two wavelengths, ${ }^{18} \lambda_{L}$ $=1.25 \mu \mathrm{m}$ gives larger $A_{S F, \max }$ than $\lambda_{L}=12.5 \mu \mathrm{m}$. By an applicative point of view, this result means that Ge-based spin-PDs are less efficient at $1550 \mathrm{~nm}$ than at $1300 \mathrm{~nm}$ (these two wavelengths correspond to the third and second windows of Si-based optical fibers employed in telecommunications). ${ }^{16}$ In the limit $l_{s f}^{S C} \gg z_{S C}$ and $l_{s f}^{S C} \gg \lambda_{L}$, instead, all the photogenerated electrons maintain their polarization, so that, in the limit case $\gamma=1$ discussed above (see Eq. (43)) the 


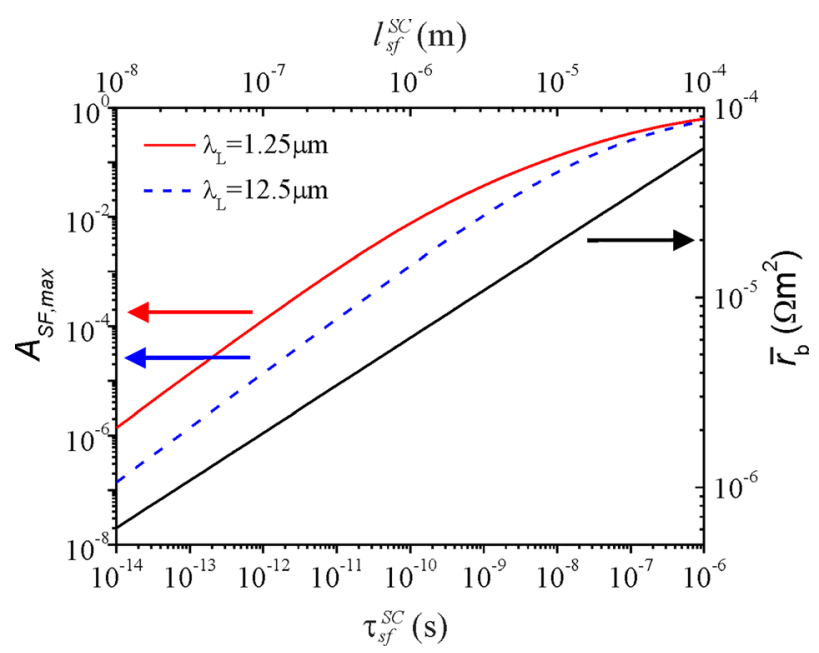

FIG. 6. Maximum figure-of-merit $A_{S F \text {, max }}$ (left scale, red and blue dashed curves) and optimal resistance barrier $\bar{r}_{B}$ (right scale, black curve) as a function of the spin relaxation time $\tau_{s f}^{S C}$ (bottom scale) and spin diffusion length $l_{s f}^{S C}$ (top scale). $A_{S F, \max }$ (see Eq. (43)) is calculated for two different light absorption lengths, $1.25 \mu \mathrm{m}$ (red line) and $12.5 \mu \mathrm{m}$ (blue dashed line). $\gamma=0.8$ is assumed.

photocurrent spin asymmetry coincides with the photogeneration spin asymmetry $\left(A_{S F, \max }=2 P_{\sigma}=\Delta P_{\sigma}\right)$.

Finally, from Fig. 5 we note that, while $A_{S F \text {, max }}$ increases with $l_{s f}^{S C}$, the area of the $\left(r_{B}, \gamma\right)$ space where $A_{S F} /$ $A_{S F}$, max is larger than a given value decreases (for example, looking at the fraction area of the $\left(r_{B}, \gamma\right)$ space where $A_{S F} /$ $A_{S F, \max }>0.25$, we find $30 \%, 21 \%$, and $13 \%$ for $l_{s f}^{S C} \sim 0.1 \mu \mathrm{m}$, $1 \mu \mathrm{m}$, and $10 \mu \mathrm{m}$, respectively). The increase of $A_{S F \text {, max }}$ with $\tau_{s f}^{S C}\left(l_{s f}^{S C}\right)$ is then counterbalanced by a stronger localization (in terms of the space of parameters) of $A_{S F}$, that is there is a smaller $\left(r_{B}, \gamma\right)$ space where to work in order to obtain $A_{S F}$ values close to its maximum.

\section{EXPERIMENTAL VALIDATION ON GERMANIUM SPIN PHOTODIODES}

In order to validate the model discussed above, we briefly report on the experimental results obtained by our group on spin-PDs based on the $\mathrm{Fe} / \mathrm{MgO} / \mathrm{Ge}$ heterostructure. ${ }^{15}$

In addition to allowing spin manipulation ${ }^{64}$ and spin transport over micrometric distances, ${ }^{36}$ with spin lifetimes in the nanosecond range, ${ }^{32,65}$ the peculiarity of $\mathrm{Ge}$ in spinoptoelectronic is the opportunity of operating the spin-PD in a wider spectral range than GaAs, from the visible to the near-infrared $(0.8 \mathrm{eV})$, including the second and the third windows of Silica optical fibers that would be not accessible to GaAs based devices, due to the much larger bandgap $(1.42 \mathrm{eV})$. Moreover, with respect to their GaAs counterparts, ${ }^{8,9}$ even employing more complex features such as quantum wells, ${ }^{10,11}$ our Ge-based spin-PDs show larger conversion efficiencies at room temperature (about $5 \%$ at $0.4 \mathrm{~V}$ bias and $1300 \mathrm{~nm}$ excitation energy, as shown in Fig. 7 and Ref. 15 , to be compared with maximum values of about $1 \%$ for GaAs-based devices reported in the literature).

Several actions could be taken for further improving the conversion efficiency $A_{S F}$. According to the work of Bottegoni

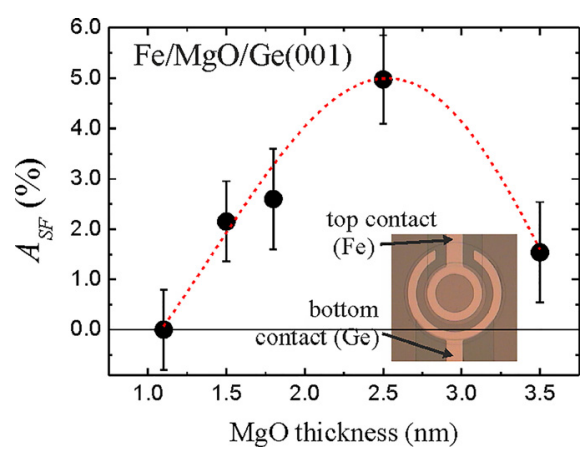

FIG. 7. Measured conversion efficiency $\left(A_{S F}\right)$ as a function of the barrier thickness $\left(t_{B}\right)$ in $\mathrm{Fe} / \mathrm{MgO} / \mathrm{Ge}$ spin-photodiodes (adapted from Ref. 15). The red dashed line is only a guide for the eyes. In the inset is reported the top view of a $\mathrm{Fe} / \mathrm{MgO} / \mathrm{Ge}$ photodiode, with indicated the top contact $(\mathrm{Fe})$ and the bottom contact $(\mathrm{Ge})$. The electrodes have annular shape, and the illuminated area is the circular region inside the circular ring of the top contact.

et al., ${ }^{49}$ a compressive strain on Ge removes the heavy-hole light-hole degeneracy leading to an increase of the polarization (a sufficient strain could achieve a $100 \%$ optical spin orientation at the band gap). Being $A_{S F}$ proportional to $P_{\sigma}$ (see Eq. (40)), this would double, in the best condition, the conversion efficiency of the device. Tang et al., ${ }^{66}$ instead, predicted that a strain along the Ge [111] direction can partially remove the degeneracy of the L valleys, leading to a reduced intervalley phonon-assisted scattering and hence to an increase of even an order of magnitude in the spin lifetime of carriers $\tau_{s f}^{S C}$. This achievement would increase the figure of merit, according to Sec. IV C. Finally, in Sec. IV and Ref. 15, we demonstrated the importance of the spatial distribution of polarized carriers with respect to the position of the $\mathrm{Fe} / \mathrm{MgO}$ detector, essentially depending on the ratio between light absorption length and spin diffusion length. Heterostructures engineering can be used to design Ge-based spin-PDs where photo-carrier generation takes place very close to the $\mathrm{MgO}$ barrier, so that the spin depolarization during transport towards the barrier becomes negligible.

The sample preparation has been reported in detail elsewhere. ${ }^{43,44} \mathrm{MgO}$ and Fe were deposited in-situ by Molecular Beam Epitaxy (MBE) on low n-doped Ge(001) commercial wafers (resistivity $\sim 47 \Omega \mathrm{cm}$ ) at room temperature $(\mathrm{RT})$ and post-annealed at $770 \mathrm{~K}$ and $470 \mathrm{~K}$, respectively, to achieve good epitaxy with limited interdiffusion at the interfaces. The Fe thickness was fixed at $10 \mathrm{~nm}$, while different thicknesses of $\mathrm{MgO}$ have been tested, ranging from $1 \mathrm{~nm}$ to $3.5 \mathrm{~nm}$. Finally, the samples were capped with $2 \mathrm{~nm}$ of $\mathrm{Au}$ in order to protect the topmost Fe layer from oxidation. SpinPDs with circular shape and different areas (from $1 \times 10^{4}$ to $5 \times 10^{5} \mu \mathrm{m}^{2}$ ) have been fabricated by means of optical lithography and ion beam etching. A top view of a spin-PD is shown in the inset of Fig. 7. Circularly polarized light, with a wavelength of $1300 \mathrm{~nm}(h \nu=0.96 \mathrm{eV}$, close to the Ge gap at the $\Gamma$ point), impinges on the device from the top and perpendicularly to the spin-PD surface. An external electromagnet was employed to provide a magnetic field perpendicular to the surface, in order to drive the Fe magnetization 
$(M)$ out-of-plane and hence parallel to the photon angular momentum. The curve reported in Fig. 7 was obtained with the electrical setup shown in Fig. 1(b) by using a lock-in technique, in order to improve the signal-to-noise ratio and to isolate the photocurrent contribution. Circularly polarized light was produced by a laser diode with a wavelength of $1300 \mathrm{~nm}(h \nu=0.96 \mathrm{eV})$, followed by a linear polarizer and a photoelastic modulator (PEM), working at $f=50 \mathrm{kHz}$ frequency. The helicity-dependent photocurrent $\left(\Delta I^{\sigma}(f)=I^{\sigma=-1}\right.$ $-I^{\sigma=+1}$ ) was converted into a proportional voltage signal by the operational amplifier and then demodulated by the lock-in amplifier. The photocurrent $I_{p h}$ was measured employing light with linear polarization, with the very same intensity of circularly polarized light. Further details on the experimental setup and measurement procedures are reported in Refs. 14-16.

The measurements have been performed in the CVMM mode (see Secs. III C and IV), applying a fixed voltage $\Delta V=0.4 \mathrm{~V}$ in order to drive the electrons from $\mathrm{Ge}$ to $\mathrm{Fe}$. In Fig. 7 is reported the conversion efficiency $A_{S F}$ vs. the $\mathrm{MgO}$ barrier thickness $t_{B}$ (red dots), measured by characterizing batches of spin-PDs with different $t_{B}$ in the range of $1 \mathrm{~nm}-3.5 \mathrm{~nm}$. A clear maximum in $A_{S F}$ is present for a barrier thickness $t_{B}=2.5 \mathrm{~nm}$, while for thinner and thicker barriers the spin filtering effect essentially vanishes. Note that the $\mathrm{MgO}$ thickness range considered in this work is $1.5 \mathrm{~nm}-3.5 \mathrm{~nm}$, that is $t_{B} \ll z_{S C}$ and $t_{B} \ll z_{F}$, so that the model, that assumes a zero-thickness barrier, can be considered valid. Obviously, the barrier thickness determines the barrier resistance $\left(r_{B}\right)$ : we do not have a direct measure of it because of the series resistance due to the highly resistive Ge substrate, so that we can only set an upper limit (5 $\times 10^{-5} \Omega \mathrm{m}^{2}$ ), equal to the substrate resistance. ${ }^{15}$ As discussed in Ref. 15, ab-initio calculations give $\gamma \sim 0.5$ for the electron tunneling asymmetry across $\mathrm{MgO}$ at the L-point in the Ge band-structure, where tunneling takes place. Coming to the model prediction, in Fig. 3 there is actually a maximum at $r_{B} \sim 5 \times 10^{-6} \Omega \mathrm{m}^{2}$ for $\gamma=0.35$, in good agreement with the presence of the experimental peak of $A_{S F}$ at $t_{B}=2.5 \mathrm{~nm}$. This result is a clear validation of the model reliability, in terms of predicting the data set $\left(r_{B}, \gamma\right)$ that maximizes $A_{S F}$.

We note that, even if the prediction of the magnitude of $A_{S F}$ is underestimated by the model (the latter calculates a value five times smaller than experimental values), probably because of oversimplifications (e.g., neglecting band bending effects, as in the FJ work) and uncertainties in parameters (barrier resistance and spin selectivity, spin diffusion length,...), we should stress that the predicted parameter dependences ( $A_{S F}$ vs. barrier thickness ${ }^{15}$ and $A_{S F}$ vs. photon energy ${ }^{18}$ ) have been fully confirmed experimentally. We can then conclude that we have been able to obtain a quite simple and, above all, analytically solvable model that could be of practical use for first-order approximation design of spin-PDs and data interpretation. More complete, and complex, models should likely improve the precision of our results, even we do not expect a relevant improvement in the comprehension of the parameter $\left(r_{B}, \gamma, l_{s f}^{S C}, \lambda_{L}\right)$ influence on the device performance.

\section{CONCLUSIONS}

In this paper, we have presented a theoretical model for the helicity-dependent spin injection of a photocurrent in a MOS-based spin photodiode. The model is based on the Fert-Jaffrès framework, ${ }^{23}$ with the introduction of the photogeneration term in the continuity equation. The model leads to the expression $\Delta V=r_{0} J_{0}+r_{\sigma} J_{p h}$, where $\Delta V$ is the voltage drop across the device, $J_{0}$ is the dc current, $J_{p h}$ is the photocurrent, $r_{0}$ is a fixed resistance per unit area, and $r_{\sigma}$ is a helicity dependent resistance per unit area, containing the dependence on the circular polarization $(\sigma)$ of the photocurrent. We defined the figure of merit of the spin photodiode as the difference, measured with the voltage $\Delta V$ fixed, between the electron current when the device is illuminated by right $(\sigma=+1)$ and left $(\sigma=-1)$ circularly polarized light, divided by the photocurrent

$$
A_{S F}=\frac{r_{S C}\left(\gamma r_{B}+\beta r_{F M}\right)}{\left(r_{F M}+r_{S C}+r_{B}\right)\left[\bar{r}_{S C}+\left(1-\beta^{2}\right) \bar{r}_{F M}\right]+\left(1-\gamma^{2}\right) r_{B}^{2}+\left(1-2 \gamma \beta+\beta^{2}\right) r_{B} r_{F M}+r_{B} r_{S C}+\beta^{2} r_{S C} r_{F M}} \times \frac{l_{s f}^{S C}}{\lambda_{L}+l_{s f}^{S C}} 2 P_{\sigma} .
$$

This expression exploits the dependence on many parameters, such as the resistance $\left(r_{B}\right)$ and spin asymmetry $(\gamma)$ of the barrier, and the resistivity $\left(\rho_{S C}\right)$, the spin diffusion length $\left(l_{s f}^{S C}\right)$, and the light absorption length $\left(\lambda_{L}\right)$ of the semiconductor. While $A_{S F}$ increases monotonically with $\gamma$, the dependence on $r_{B}$ is more complicated: $A_{S F}$ is minimum for $r_{B}=0$ and $r_{B} \gg \rho_{S C} l_{s f}^{S C}$, while the maximum is attained at

$$
\overline{r_{B}}=\rho_{S C} \sqrt{\frac{z_{S C} l_{s f}^{S C}}{1-\gamma^{2}}}
$$

This formula allows us to find the optimal parameter set for maximizing $A_{S F}$, which represents the real efficiency of the spin-PD as a polarimeter. We stress the fact that, at variance with the models reported in the literature until now, our model can be analytically solved, leading to simple mathematical formulae, and in this sense it can be practically employed for data interpretation and first-approximation device engineering of spin-PDs. An experimental validation of Eq. (52) is reported for the case of $\mathrm{Fe} / \mathrm{MgO} / \mathrm{Ge}$ spinphotodiodes, where the relationship between the barrier thickness and the conversion efficiency $A_{S F}$ is interpreted in the frame of our model.

\section{ACKNOWLEDGMENTS}

The authors wish to thank R. Bertacco, D. Petti, G. Isella, F. Pezzoli, and A. Giorgioni for valuable discussions. 
They gratefully acknowledge financial support from Fondazione Cariplo via the projects Search IV (Grant No. 2013-0623), Magister (Grant No. 2013-0726), and from Italian Ministry of Research via the project FIRB OSSIDI NANOSTRUTTURATI: MULTI-FUNZIONALITA' E APPLICAZIONI (RBAP115AYN). This work was partially performed at Polifab, the micro and nanofabrication facility of Politecnico di Milano.

\section{APPENDIX A: THE CONTINUITY EQUATION IN THE SEMICONDUCTOR}

The continuity equations for the semiconductor (see Ref. 67) are

$$
\begin{aligned}
& \frac{\partial n}{\partial t}=G_{n}-U_{n}+\frac{1}{q} \nabla \cdot \vec{J}_{n}, \\
& \frac{\partial p}{\partial t}=G_{p}-U_{p}-\frac{1}{q} \nabla \cdot \vec{J}_{p} .
\end{aligned}
$$

$G_{n}$ and $G_{p}$ are the electron $(n)$ and hole $(p)$ generation rates related to photo-generation. $U_{n}$ and $U_{p}$ are the corresponding net recombination rates, taking into account the band-toband electron-hole recombination and the thermal generation. Under the assumption of low-level injection (verified in our system, being $J_{p h} / J_{0} \sim 0.1 \%$, as shown in Sec. IV), the net recombination rates are proportional to the excess (with respect to thermal equilibrium) carrier densities $\Delta n$ and $\Delta p$ : $U_{n}=\Delta n / \tau_{n}$ and $U_{p}=\Delta p / \tau_{p}$, where $\tau_{n}$ and $\tau_{p}$ are the electronic carrier lifetimes. Note that the recombination channel is always present and turns out to be fundamental for measuring a contrast between current densities for illumination by opposite light helicities.

A simple example can explain the role of recombination. Let us consider a fully circularly polarized light inducing fully spin polarized electrons by optical spin orientation in a system with a very good spin filtering of the barrier (barrier selectivity $\gamma$ close to 1 ). For a spin up population, the transmission through the barrier will be high and, consequently, the accumulation at the $\mathrm{MgO} / \mathrm{Ge}$ interface will be relatively low. On the contrary, a reversal of the light helicity will produce a spin down population with low transmission through the $\mathrm{MgO}$ barrier. These will lead to a significant accumulation at the $\mathrm{MgO} / \mathrm{Ge}$ interface. In steady state conditions and in the absence of a recombination channel, the current crossing the barrier will be the same for right or left circularly polarized light, because all the carriers must cross the $\mathrm{MgO}$ barrier. Then, a recombination channel must be present to take into account the different accumulation of spin up and spin down electrons at the interface and to "switch on" a helicity-dependent photocurrent. A spin down polarized population will produce high accumulation and high recombination, while for a spin-up population the accumulation will be negligible and thus the recombination has a minor role, leading to a larger photocurrent.

Note that recombinations at the $\mathrm{MgO} / \mathrm{Ge}$ interface may occur, but their effect is qualitatively similar to bulk Ge band-to-band recombinations, so it can be omitted for sake of simplicity and included in the net recombination rates $\left(U_{n}\right.$ and $U_{p}$ ). Moreover, note that the low density of interface states and defects at the interface between $\mathrm{MgO}$ and $\mathrm{Ge}$, indicated by the effective Fermi level depinning, ${ }^{42}$ reduces the relative weight of this effect for the system.

As discussed above, within the low-level injection regime, the net recombination rates are proportional to the excess carriers only, in first approximation $\left(U_{n}=\Delta n / \tau_{n}\right.$, $\left.U_{p}=\Delta p / \tau_{p}\right){ }^{67}$ These excess carriers $\Delta n$ and $\Delta p$ come from the photo-generation, i.e., from the light absorption populating the valence band (conduction band) with photo-excited holes (electrons). It follows that the generation rates $G_{n}$ and $G_{p}$ are proportional to $\Delta n$ and $\Delta p$, respectively, and we can assume the same spatial profile for generation and recombination, so to define the effective "combined" rates, including both photogeneration and recombination

$$
\begin{aligned}
& G_{e f f, n}=G_{n}-U_{n}, \\
& G_{e f f, p}=G_{p}-U_{p} .
\end{aligned}
$$

In steady-state conditions, we have $\partial n / \partial t=0$ and $\partial p / \partial t=0$, so that Eqs. (A1) and (A2) become

$$
\begin{aligned}
& G_{e f f, n}+\frac{1}{q} \nabla \cdot J_{n}=0, \\
& G_{e f f, p}-\frac{1}{q} \nabla \cdot J_{p}=0 .
\end{aligned}
$$

Note that, because of the charge neutrality of both photogeneration and recombination, the effective generation rates for holes and electrons are equal $\left(G_{\text {eff }, n}=G_{e f f, p}\right)$. In steady state conditions, Eqs. (A5) and (A6) give a total current (electrons + holes) constant along the device length: $\nabla \cdot J_{n}+\nabla \cdot J_{p}=\nabla \cdot\left(J_{n}+J_{p}\right)=\nabla \cdot J_{\text {tot }}=0$.

In our model, we consider electrons only, that is Eqs. (A1), (A3), and (A5). In our device geometry, $\vec{J}_{n}$ is directed along $z$ and $G_{n}$ and $U_{n}$ depend only on the $z$-coordinate. Eq. (A5) corresponds to the steady state version of Eq. (5) in the manuscript, summing up the + and - electrons (with $\left.\tau_{s f}^{+}=\tau_{s f}^{-}\right) \quad$ and assuming $G_{e f f, n}^{+(-)}=G_{n}^{+(-)}(z)-U_{n}^{+(-)}(z)$ $=\eta_{\sigma}^{+(-)} \exp \left(-\alpha_{L} z\right)$ (Eq. (4) in the manuscript). Note that a more sophisticated model should take into account explicitly the recombination time of electrons that plays a key role in the observation of a non zero $A_{S F}$ as discussed above (in the absence of such a channel, or equivalently in the case of an infinite recombination time, in steady state conditions spin up and down currents will be the same, independently on their different accumulation at the $\mathrm{MgO} / \mathrm{Ge}$ interface, so that $A_{S F}$ would be identically zero), leading to an expression of $A_{S F}$ depending on it.

The exponential decay of $G_{n}^{+(-)}$is due to the exponential attenuation of light in the semiconductor, coming from the classical theory of electromagnetic waves absorbed in a medium with complex refractive index. The recombination of electrons $U_{n}^{+(-)}$can be considered, as a first approximation, an exponential too, with the same spatial profile of the photo-generation, being $U_{n}=\Delta n / \tau_{n}$ (see Ref. 67) and thus proportional to the number of photogenerated electrons. 
Eq. (A5) applies everywhere in the semiconductor and states that in steady state conditions (as those considered in our paper) the divergence of $\vec{J}_{n}$ is not zero but depends on the effective generation rate

$$
\nabla \cdot \vec{J}_{n}=-q G_{e f f, n} .
$$

Applying the divergence theorem to Eq. (A7) on a volume $V$ with surface boundary $\Sigma$, we obtain

$$
\int_{\Sigma} \vec{J}_{n} \cdot \vec{n} d \Sigma=-q \int_{V} G_{e f f, n} d V
$$

where $\vec{n}$ is the normal unit vector pointing outward the surface element $d \Sigma$.

We consider a volume $V$ shaped as a cylinder with base area $A$, parallel to the $x y$ plane, and extending in the $z$-direction from $z=0$ to $z=z_{\mathrm{SC}}$. This semiconductor geometry is the same employed in our device. Eq. (A8) becomes

$$
\begin{aligned}
J_{n}\left(z_{S C}\right) A-J_{n}(0) A & =-q \int_{0}^{z_{S C}} G_{e f f, n} A d z \\
& =-q \int_{0}^{z_{S C}}\left(\eta_{\sigma}^{+}+\eta_{\sigma}^{-}\right) \exp \left(-\alpha_{L} z\right) A d z \\
& =q \frac{\left(\eta_{\sigma}^{+}+\eta_{\sigma}^{-}\right)}{\alpha_{L}} A .
\end{aligned}
$$

$J_{n}(0)$ is the electron current density at the interface between the semiconductor and the ferromagnet. Because in the latter there is no electron generation or recombination, the divergence is zero $\left(\nabla \cdot \vec{J}_{n}=0\right)$ and thus $J_{n}(0)=J_{0}$, where $J_{0}$ is the electron current density in the ferromagnet as defined in the paper. $J_{n}\left(z_{S C}\right)$ is the electron current density at the other side of the semiconductor, called in the Paper $\bar{J}$. Defining $\bar{\eta}_{\sigma}=\eta_{\sigma}^{+}+\eta_{\sigma}^{-}$as in the Paper and taking $q=e$, we finally obtain Eq. (19) of the Paper

$$
J_{0}+\frac{e \bar{\eta}_{\sigma}}{\alpha_{L}}=\bar{J} .
$$

\section{APPENDIX B: CALCULATION OF THE PARAMETERS}

\section{$A_{4}, B_{1}, A N D B_{2}$}

With some mathematics, Eqs. (20) and (23) in the text (the latter for + and for - electrons separately) can be rewritten as

$$
\begin{aligned}
& \frac{A_{4}}{r_{S C}}-\frac{B_{1}}{r_{F M}}=\beta e J_{0}+\beta \frac{\omega}{r_{S C}} \bar{a}_{\sigma}-\frac{\omega}{r_{S C}} \Delta a_{\sigma}, \\
A_{4}+ & {\left[\frac{(1+\beta) r_{F M}+(1+\gamma) r_{B}}{r_{F M}}\right] B_{1}-B_{2} } \\
= & (1+\gamma-\beta-\beta \gamma) r_{B} e J_{0} \\
+ & {\left[1+(1+\gamma-\beta-\beta \gamma) \omega \frac{r_{B}}{r_{S C}}\right] \bar{a}_{\sigma}-\Delta a_{\sigma}, } \\
-A_{4}+ & {\left[\frac{(-1+\beta) r_{F M}+(-1+\gamma) r_{B}}{r_{F M}}\right] B_{1}-B_{2} } \\
= & (1-\gamma+\beta-\beta \gamma) r_{B} e J_{0} \\
+ & {\left[1+(1-\gamma+\beta-\beta \gamma) \omega \frac{r_{B}}{r_{S C}}\right] \bar{a}_{\sigma}+\Delta a_{\sigma} . }
\end{aligned}
$$

Instead of Eqs. (B2) and (B3), for convenience we take their sum (Eq. (B4)) and difference (Eq. (B5)), respectively

$$
\begin{aligned}
{\left[\frac{\beta r_{F M}+\gamma r_{B}}{r_{F M}}\right] B_{1}-B_{2}=} & (1-\beta \gamma) r_{B} e J_{0} \\
& +\left[1+(1-\beta \gamma) \omega \frac{r_{B}}{r_{S C}}\right] \bar{a}_{\sigma}
\end{aligned}
$$

$A_{4}+\left[\frac{r_{F M}+r_{B}}{r_{F M}}\right] B_{1}=(\gamma-\beta) r_{B} e J_{0}+\left[(\gamma-\beta) \omega \frac{r_{B}}{r_{S C}}\right] \bar{a}_{\sigma}-\Delta a_{\sigma}$.

Eqs. (B1), (B4), and (B5) constitute a linear system of three equations in the three parameters $\mathrm{A}_{4}, \mathrm{~B}_{1}$, and $\mathrm{B}_{2}$. The solution is

$$
\begin{aligned}
A_{4}= & \frac{1}{r_{F M}+r_{S C}+r_{B}}\left\{r_{S C}\left(\gamma r_{B}+\beta r_{F M}\right) e J_{0}\right. \\
& \left.+\left(\gamma r_{B}+\beta r_{F M}\right) \omega \bar{a}_{\sigma}-\left[r_{S C}+\left(r_{B}+r_{F M}\right) \omega\right] \Delta a_{\sigma}\right\}, \quad \\
B_{1}= & \frac{r_{F M}}{r_{F M}+r_{S C}+r_{B}}\left\{\left[(\gamma-\beta) r_{B}-\beta r_{S C}\right] e J_{0}\right. \\
& \left.+\frac{(\gamma-\beta) r_{B}-\beta r_{S C}}{r_{S C}} \omega \bar{a}_{\sigma}+(\omega-1) \Delta a_{\sigma}\right\}, \\
B_{2}= & -\frac{1}{r_{F M}+r_{S C}+r_{B}} \\
& \times\left\{\left[\left(1-\gamma^{2}\right) r_{B}^{2}+\left(1-2 \gamma \beta+\beta^{2}\right) r_{B} r_{F M}+r_{B} r_{S C}\right.\right. \\
& \left.+\beta^{2} r_{S C} r_{F M}\right] e J_{0}+\frac{1}{r_{S C}}\left[\left(1-\gamma^{2}\right) r_{B}^{2}\right. \\
& \left.+\left(1-2 \gamma \beta+\beta^{2}\right) r_{B} r_{F M}+r_{B} r_{S C}+\beta^{2} r_{S C} r_{F M}\right] \omega \bar{a}_{\sigma} \\
& \left.+\left[r_{F M}+r_{S C}+r_{B}\right] \bar{a}_{\sigma}-\left(\gamma r_{B}+\beta r_{F M}\right)(\omega-1) \Delta a_{\sigma}\right\} .
\end{aligned}
$$

\section{APPENDIX C: CALCULATION OF THE OPTIMAL RESISTANCE BARRIER}

The first derivative of Eq. (41) in the variable $r_{B}$ is

$$
\begin{aligned}
\frac{\partial A_{S F}}{\partial r_{B}}= & \gamma r_{S C} \frac{\bar{r}_{S C} r_{S C}-\left(1-\gamma^{2}\right) r_{B}{ }^{2}}{\left[\bar{r}_{S C}\left(r_{S C}+r_{B}\right)+\left(1-\gamma^{2}\right) r_{B}{ }^{2}+r_{B} r_{S C}\right]^{2}} \\
& \times \frac{\omega}{1+\omega} 2 P_{\sigma} .
\end{aligned}
$$

The approximations $r_{F M} \ll r_{B}, r_{F M} \ll r_{S C}$, and $\bar{r}_{F M} \ll \bar{r}_{S C}$ have been employed, as in the text.

$A_{S F}\left(\overline{r_{B}}\right)$ presents a stationary point when Eq. (C1) is equal to zero: this happens for $r_{B}$ given by

$$
\overline{r_{B}}=\sqrt{\frac{r_{S C} r_{S C}}{1-\gamma^{2}}}=\rho_{S C} \sqrt{\frac{z_{S C} l_{s f}^{S C}}{1-\gamma^{2}}} .
$$

The second partial derivative of Eq. (41) in the variable $r_{B}$ is 


$$
\frac{\partial^{2} A_{S F}}{\partial r_{B}^{2}}=-2 \gamma r_{S C} \frac{3 r_{B} \bar{r}_{S C} r_{S C}\left(1-\gamma^{2}\right)-\left(1-\gamma^{2}\right)^{2} r_{B}^{3}+\bar{r}_{S C}^{2} r_{S C}+\bar{r}_{S C} r_{S C}{ }^{2}}{\left[\bar{r}_{S C}\left(r_{S C}+r_{B}\right)+\left(1-\gamma^{2}\right) r_{B}{ }^{2}+r_{B} r_{S C}\right]^{3}} \frac{\omega}{1+\omega} 2 P_{\sigma} .
$$

Evaluated in $\bar{r}_{B}$ given by Eq. (C2), this derivative is always negative

$$
\left.\frac{\partial^{2} A_{S F}}{\partial r_{B}^{2}}\right|_{\overline{r B}}=-2 \gamma r_{S C}^{2} \bar{r}_{S C} \frac{2\left(\bar{r}_{S C} r_{S C}\right)^{1 / 2} \sqrt{1-\gamma^{2}}+\bar{r}_{S C}+r_{S C}}{\left[\bar{r}_{S C}\left(r_{S C}+r_{B}\right)+\left(1-\gamma^{2}\right) r_{B}^{2}+r_{B} r_{S C}\right]^{3}} \frac{\omega}{1+\omega} 2 P_{\sigma}<0,
$$

$\bar{r}_{B}$ thus corresponds to a maximum of $A_{S F}$, whatever the other parameters $\left(\bar{r}_{S C}, r_{S C}, \gamma\right)$ are.

${ }^{1}$ S. Hövel, N. C. Gerhardt, M. R. Hofmann, F.-Y. Lo, D. Reuter, A. D. Wieck, E. Schuster, H. Wende, and W. Keune, Phys. Status Solidi C 6, 436 (2009).

${ }^{2}$ M. Oestreich, J. Hübner, D. Hägele, M. Bender, N. Gerhardt, M. Hofmann, W. W. Rühle, H. Kalt, T. Hartmann, P. Klar, W. Heimbrodt, and W. Stolz, Adv. Solid State Phys. 41, 173 (2001).

${ }^{3}$ H.-C. Huang, Appl. Opt. 36, 6968 (1997).

${ }^{4}$ J. A. C. Bland, T. Taniyama, W. S. Cho, and J. S. Steinmuller, Curr. Appl. Phys. 3, 429 (2003).

${ }^{5}$ R. Fiederling, M. Keim, G. Reuscher, W. Ossau, G. Schmidt, A. Waag, and L. W. Molenkamp, Nature 402, 787 (1999).

${ }^{6}$ Y. Ohno, D. K. Young, B. Beschoten, F. Matsukura, H. Ohno, and D. D. Awschalom, Nature 402, 790 (1999).

${ }^{7}$ O. M. J. van't Erve, G. Kioseoglou, A. T. Hanbicki, C. H. Li, B. T. Jonker, R. Mallory, M. Yasar, and A. Petrou, Appl. Phys. Lett. 84, 4334 (2004).

${ }^{8}$ A. Hiroata, Y. B. Xu, C. M. Guertler, and J. A. C. Bland, J. Appl. Phys. 85, 5804 (1999).

${ }^{9}$ T. Tanyiama, G. Wastlbauer, A. Ionescu, M. Tselepi, and J. A. C. Bland, Phys. Rev. B 68, 134430 (2003).

${ }^{10}$ P. Renucci, V. G. Truong, H. Jaffrès, L. Lombez, P. H. Binh, T. Amand, J. M. George, and X. Marie, Phys. Rev. B 82, 195317 (2010).

${ }^{11}$ R. Farshchi, M. Ramsteiner, J. Herfort, A. Tahraoui, and H. T. Grahn, Appl. Phys. Lett. 98, 162508 (2011).

${ }^{12}$ A. N. Chantis and D. L. Smith, Phys. Rev. B 78, 235317 (2008).

${ }^{13}$ J. D. Albrecht and D. L. Smith, Phys. Rev. B 68, 035340 (2003).

${ }^{14}$ C. Rinaldi, M. Cantoni, D. Petti, and R. Bertacco, J. Appl. Phys. 111, 07C312 (2012).

${ }^{15}$ C. Rinaldi, M. Cantoni, D. Petti, A. Sottocorno, M. Leone, N. M. Caffrey, S. Sanvito, and R. Bertacco, Adv. Mater. 24, 3037 (2012).

${ }^{16}$ C. Rinaldi, M. Espahbodi, M. Cantoni, and R. Bertacco, Proc. SPIE 8461, 84611F (2012).

${ }^{17}$ C. Rinaldi, S. Bertoli, M. Cantoni, C. Manzoni, M. Marangoni, G. Cerullo, M. Bianchi, R. Sordan, and R. Bertacco, Proc. SPIE 9167, 916709 (2014).

${ }^{18}$ C. Rinaldi, M. Cantoni, M. Marangoni, C. Manzoni, G. Cerullo, and R. Bertacco, Phys. Rev. B 90, 161304(R) (2014).

${ }^{19}$ B. Endres, M. Ciorga, M. Schmid, M. Utz, D. Bougeard, D. Weiss, G. Bayreuther, and C. H. Back, Nat. Commun. 4, 2068 (2013).

${ }^{20}$ R. Ginige, B. Corbett, M. Modreanu, C. Barrett, J. Hilgarth, G. Isella, D. Chrastina, and H. von Kanel, Semicond. Sci. Technol. 21, 775 (2006).

${ }^{21}$ M. M. Oye, D. Shahrjerdi, I. Ok, J. B. Hurst, S. D. Lewis, S. Dey, D. Q. Kelly, S. Joshi, T. J. Mattord, X. Yu, M. A. Wistey, J. S. Harris, Jr., A. L. Holmes, Jr., J. C. Lee, and S. K. Banerjee, J. Vac. Sci. Technol., B 25, 1098 (2007).

${ }^{22}$ I. Žutic, J. Fabian, and S. Das Sarma, Rev. Mod. Phys. 76, 323 (2004).

${ }^{23}$ A. Fert and H. Jaffrès, Phys. Rev. B 64, 184420 (2001).

${ }^{24}$ Y. Lu, J. C. Le Breton, P. Turban, B. Lépine, P. Schieffer, and G. Jézéquel, Appl. Phys. Lett. 89, 152106 (2006).

${ }^{25}$ T. Valet and A. Fert, Phys. Rev. B 48, 7099 (1993).

${ }^{26}$ D. T. Pierce, R. J. Celotta, G.-C. Wang, W. N. Unertl, A. Galejs, C. E. Kuyatt, and S. R. Mielczarek, Rev. Sci. Instrum. 51, 478 (1980).

${ }^{27}$ I. Žutić, J. Fabian, and S. Das Sarma, Phys. Rev. B 64, 121201 (2001).

${ }^{28}$ M. E. Flatté and J. M. Byers, Phys. Rev. Lett. 84, 4220 (2000).

${ }^{29}$ M. Jullière, Phys. Lett. A 54, 225 (1975).

${ }^{30}$ D. T. Pierce and F. Meier, Phys. Rev. B 13, 5484 (1976).

${ }^{31}$ J. Rioux and J. E. Sipe, Phys. Rev. B 81, 155215 (2010).
${ }^{32}$ F. Pezzoli, F. Bottegoni, D. Trivedi, F. Ciccacci, A. Giorgioni, P. Li, S. Cecchi, E. Grilli, Y. Song, M. Guzzi, H. Dery, and G. Isella, Phys. Rev. Lett. 108, 156603 (2012).

${ }^{33}$ Because linearly polarized light is equivalent to the combination of left and right circularly polarized lights with the same intensity, the net degree of optical spin orientation of the photo-generated carriers will be zero.

${ }^{34}$ S. J. Steinmuller, C. M. Gürtler, G. Wastlbauer, and J. A. C. Bland, Phys. Rev. B 72, 045301 (2005)

${ }^{35}$ E.-S. Liu, J. Nah, K. M. Varahramyan, and E. Tutuc, Nano Lett. 10, 3297 (2010).

${ }^{36}$ C. Shen, T. Trypiniotis, K. Y. Lee, S. N. Holmes, R. Mansell, and M. Husain, Appl. Phys. Lett. 97, 162104 (2010).

${ }^{37}$ D. Lee, S. Raghunathan, R. Wilson, D. E. Nikonov, K. Saraswat, and S. X. Wang, Appl. Phys. Lett. 96, 052514 (2010).

${ }^{38}$ Y. Zhou, M. Ogawa, M. Bao, W. Han, R. K. Kawakami, and K. L. Wang, Appl. Phys. Lett. 94, 242104 (2009).

${ }^{39}$ Y. Zhou, W. Han, Y. Wang, F. Xiu, J. Zou, R. K. Kawakami, and K. L. Wang, Appl. Phys. Lett. 96, 102103 (2010).

${ }^{40}$ K.-R. Jeon, B.-C. Min, H.-S. Lee, I.-J. Shin, C.-Y. Park, and S.-C. Shin, Appl. Phys. Lett. 97, 022105 (2010)

${ }^{41}$ Y. Zhou, W. Han, L.-T. Chang, F. Xiu, M. Wang, M. Oehme, I. A. Fischer, J. Schulze, R. K. Kawakami, and K. L. Wang, Phys. Rev. B 84, 125323 (2011).

${ }^{42}$ M. Cantoni, D. Petti, C. Rinaldi, and R. Bertacco, Appl. Phys. Lett. 98, 032104 (2011).

${ }^{43}$ D. Petti, M. Cantoni, C. Rinaldi, S. Brivio, R. Bertacco, J. Gazquez, and M. Varela, J. Appl. Phys. 109, 084909 (2011).

${ }^{44}$ M. Cantoni, D. Petti, C. Rinaldi, and R. Bertacco, Microelectron. Eng. 88, 530 (2011).

${ }^{45}$ D. Petti, M. Cantoni, C. Rinaldi, and R. Bertacco, J. Phys.: Conf. Ser. 292, 012010 (2011).

${ }^{46}$ J. Gazquez, M. Varela, D. Petti, M. Cantoni, C. Rinaldi, S. Brivio, and R. Bertacco, J. Mater. Sci. 46, 4157 (2011).

${ }^{47}$ We note that, according to calculations reported in Ref. 31, the polarization presents a very slow energy dependence between 0.8 and $1 \mathrm{eV}$, so that we can reasonably assume $P_{\sigma}=0.5$ at $0.95 \mathrm{eV}$. In general, both $P_{\sigma}$ and $\lambda_{L}$, and consequently $F$, depend on the photon energy, but the study of this dependence is beyond the scope of this paper and has been reported elsewhere by the authors (Ref. 18).

${ }^{48}$ R. Allenspach, F. Meier, and D. Pescia, Phys. Rev. Lett. 51, 2148 (1983).

${ }^{49}$ F. Bottegoni, G. Isella, S. Cecchi, and F. Ciccacci, Appl. Phys. Lett. 98, 242107 (2011).

${ }^{50}$ F. Pezzoli, L. Qing, A. Giorgioni, G. Isella, E. Grilli, M. Guzzi, and H. Dery, Phys. Rev. B 88, 045204 (2013).

${ }^{51}$ S. Patibandla, G. M. Atkinson, S. Bandyopadhyay, and G. C. Tepper, Physica E 42, 1721 (2010).

${ }^{52}$ P. P. Debye and E. M. Conwell, Phys. Rev. 93, 693 (1954).

${ }^{53}$ H. R. Philipp and E. A. Taft, Phys. Rev. 113, 1002 (1959).

${ }^{54}$ C. H. Park, B. C. Lee, and J. I. Lee, J. Korean Phys. Soc. 47, 655 (2005).

${ }^{55}$ T. L. Monchesky, A. Enders, R. Urban, K. Myrtle, B. Heinrich, X.-G. Zhang, W. H. Butler, and J. Kirschner, Phys. Rev. B 71, 214440 (2005).

${ }^{56}$ We note that the barrier selectivity $\gamma$ we employed $(0.35)$ has been predicted (see Ref. 15) considering a $\mathrm{Fe} / \mathrm{MgO} / \mathrm{Ge}$ structure with an ultrathin $\mathrm{MgO}$ barrier (less than $1.1 \mathrm{~nm}$ ) and assuming that all electrons tunnel from the $\mathrm{L}$ point, after full relaxation from the photogeneration point in $\Gamma$. Electrons photogenerated closer to the barrier could anyway experience tunneling directly from $\Gamma$, where the barrier selectivity $\gamma$ has been predicted to be very high (close to 1). In this case, the real value of $\gamma$ could be significantly higher than the theoretical one calculated and employed in this paper. 
${ }^{57}$ With reference to the inset of Fig. 7 or to Fig. 1 of Ref. 14, the distance between the inner ring (top contact on $\mathrm{Fe}$ ) and the outer ring (bottom contact on $\mathrm{Ge}$ ) is $20 \mu \mathrm{m}$, so that the effective distance travelled by electrons from the $\mathrm{MgO}$ barrier to the bottom contact on $\mathrm{Ge}$ is equal (or larger) than this value. The assumptions $z_{S C} \gg l_{s f}^{S C}$ and $z_{S C} \gg \lambda_{L}$ are then fully satisfied.

${ }^{58}$ Z. G. Yu and M. E. Flatté, Phys. Rev. B 66, 201202(R) (2002).

${ }^{59} \mathrm{~A}$ general rule for operating in the low-field regime $\left(E<E_{\mathrm{C}}\right)$ is $\rho_{S C}\left(J_{0}+J_{p h}\right)<k_{B} T / e l_{s f}^{S C}$ : this imposes a limit on the maximum current flowing in the device: $J_{0}+J_{p h}<k_{B} T / e \rho_{S C} l_{s f}^{S C}=k_{B} T / e r_{S C}$. In our system, this means $J_{0}+J_{p h}<5.41 \times 10^{4} \mathrm{~A} / \mathrm{m}^{2}$, corresponding to $I_{0}+I_{p h}<24$ $\mathrm{mA}$ and $\Delta V \approx r_{0} J_{0}<0.75 \mathrm{~V}$ (recalling that $J_{p h} \ll J_{0}$ ).

${ }^{60}$ The $\gamma=0$ case is not physically meaningful because, due to the different density of states at the Fermi level for spin up and spin down electrons in the ferromagnet, the relative tunneling transmission probabilities will be intrinsically different, so that $\gamma>0$.

${ }^{61}$ G. Schmidt, D. Ferrand, L. W. Molenkamp, A. T. Filip, and B. J. van Wees, Phys. Rev. B 62, R4790 (2000).

${ }^{62}$ E. Rashba, Phys. Rev. B 62, R16267 (2000).

${ }^{63}$ W. C. Dash and R. Newman, Phys. Rev. 99, 1151 (1955).

${ }^{64}$ S. D. Ganichev, S. N. Danilov, V. V. Bel'kov, S. Giglberger, S. A. Tarasenko, E. L. Ivchenko, D. Weiss, W. Jantsch, F. Schäffler, D. Gruber, and W. Prettl, Phys. Rev. B 75, 155317 (2007).

${ }^{65}$ A. Giorgioni, E. Vitiello, E. Grilli, M. Guzzi, and F. Pezzoli, Appl. Phys. Lett. 105, 152404 (2014).

${ }^{66}$ J. M. Tang, B. T. Collins, and M. E. Flatté, Phys. Rev. B 85, 045202 (2012).

${ }^{67}$ S. M. Sze and K. K. Ng, Physics of Semiconductor Devices, 3rd ed. (Wiley-Interscience, 2006). 